\title{
The background field method and critical vector models
}

\author{
Mikhail Goykhman, ${ }^{a}$ Vladimir Rosenhaus ${ }^{b, c, 1}$ and Michael Smolkin ${ }^{a}$ \\ ${ }^{a}$ The Racah Institute of Physics, The Hebrew University of Jerusalem, \\ Jerusalem 91904, Israel \\ ${ }^{b}$ School of Natural Sciences, Institute for Advanced Study, \\ 1 Einstein Drive, Princeton, NJ 08540, U.S.A. \\ ${ }^{c}$ Initiative for the Theoretical Sciences, The Graduate Center, City University of New York, \\ 365 Fifth Ave, New York, NY 10016, U.S.A. \\ E-mail: michael.goykhman@mail.huji.ac.il, vladr@ias.edu, \\ michael.smolkin@mail.huji.ac.il
}

ABSTRACT: We use the background field method to systematically derive CFT data for the critical $\phi^{6}$ vector model in three dimensions, and the Gross-Neveu model in dimensions $2 \leq d \leq 4$. Specifically, we calculate the OPE coefficients and anomalous dimensions of various operators, up to next-to-leading order in the $1 / N$ expansion.

Keywords: 1/N Expansion, Conformal Field Theory, Nonperturbative Effects, Field Theories in Lower Dimensions

ARXIV EPRINT: 2009.13137

\footnotetext{
${ }^{1}$ On leave from: Initiative for the Theoretical Sciences, The Graduate Center, City University of New York, 365 Fifth Ave, New York, NY 10016, U.S.A.
} 


\section{Contents}

1 Introduction 1

2 Review of the $\phi^{6}$ model $\quad 2$

2.1 UV fixed point 5

2.2 Anomalous dimensions of $\phi$ and $\phi^{2} \quad 6$

3 CFT data for the critical $\phi^{6}$ model 9

3.1 Anomalous dimensions of $\left(\phi^{2}\right)^{n} \quad 9$

$\begin{array}{lll}3.2 & \text { OPE coefficients } & 12\end{array}$

4 Review of the Gross-Neveu model $\quad 14$

$\begin{array}{lll}4.1 & \text { Feynman rules and useful identities } & 14\end{array}$

$\begin{array}{ll}4.2 \text { Propagators at the next-to-leading order } & 16\end{array}$

$\begin{array}{lll}4.3 & \text { Gross-Neveu-Yukawa model } & 18\end{array}$

5 CFT data for the critical Gross-Neveu model 20

6 Discussion $\quad 26$

$\begin{array}{ll}\text { A Interaction vertex in the critical } \phi^{4} \text { model } & 27\end{array}$

B The $\phi^{6}$ model in terms of auxiliary fields 31

\section{Introduction}

Due to their theoretical and experimental importance, conformal field theories (CFTs) have been extensively studied over the decades, through a broad range of techniques. One of the most prominent is the conformal bootstrap [1-5] which, rather than using a specific Lagrangian, works with general axiomatic parameters, such as the scaling dimensions of local operators and their operator product expansion (OPE) coefficients. The focus of this paper is inspired by the bootstrap program, but the CFT data is calculated using a complementary method: the diagrammatic $1 / N$ expansion in the context of critical vector models $[6,7]$.

The $1 / N$ expansion is particularly useful when the theory is strongly coupled and the corresponding CFT cannot be studied perturbatively $[8,9]$. Thus, for instance, it provides an alternative to the classic $\epsilon$-expansion, originally formulated for the scalar field theory with a quartic interaction in $4-\epsilon$ dimensions [10]. ${ }^{1}$ Moreover, certain non-renormalizable quantum field theories which are not tractable within a traditional perturbative expansion

\footnotetext{
${ }^{1}$ The Banks-Zaks fixed point is another example of a perturbatively tractable CFT [11, 12].
} 
become analytically controllable in the large $N$ framework. These include the most wellknown vector models with a quartic interaction: the $\phi^{4} O(N)$ vector model for bosons [13], and the Gross-Neveu model for fermions $[14,15]$. Another interesting example in this class of models is the cubic scalar vector model in $6-\epsilon$ dimensions $[16-18] .{ }^{2}$

A vector model which has been far less studied is the sextic $\phi^{6} O(N)$ model, in three dimensions [26], for recent studies see [27, 28]. The model has the notable feature - which motivated our study of it - of having a UV conformal fixed point, at large $N$, directly in three dimensions. An $\epsilon$ expansion, as for the Wilson-Fisher fixed point in the quartic model, is not required. In this paper we compute various anomalous dimensions and conformal three-point functions in the sextic $O(N)$ vector model, up to next-to-leading order in $1 / N$.

In the process of this study we found that, while the anomalous dimensions in critical vector models have been extensively studied, there has been much less study of the conformal three-point functions (the OPE data). We find that our method of computation - in particular, applying the background field method [29] in the context of large $N$ conformal perturbation theory - provides a simple and coherent framework. We apply this method to the standard vector models, extending known results in the literature.

In section 2 we review the $\phi^{6}$ vector model, the location of the UV fixed point at large $N$, and the anomalous dimensions of the field. Then in section 3 we compute several conformal three-point functions at next-to-leading order in $1 / N$. In section 4 we review the Gross-Neveu model, and in section 5 we compute several conformal three-point functions at next-to-leading order in $1 / N$. In appendix $\mathrm{A}$ we perform similar computations in the context of the quartic $O(N)$ vector model. In appendix B we discuss the $\phi^{6}$ model, when the field $\phi$ is integrated out.

\section{Review of the $\phi^{6}$ model}

Consider the following three-dimensional $O(N)$ vector model with sextic interaction,

$$
S=\int d^{3} x\left(\frac{1}{2}(\partial \phi)^{2}+\frac{g_{6}}{6 N^{2}}\left(\phi^{2}\right)^{3}\right) .
$$

The beta function of $g_{6}$ vanishes identically in the large $N$ limit, and the model exhibits a line of fixed points, $0 \leq g_{6} \leq(4 \pi)^{2}$, where the boundaries of the conformal window are found by demanding stability of the effective potential [30], see also [31, 32]. The $1 / N$ corrections lift this degeneracy and only one isolated UV fixed point, which lies outside the stability region, survives at finite $N[26]$. The instability effect is, however, nonperturbative in $1 / N$. Moreover, it is not clear whether it will persist at any finite $N$, see [33] and references therein for a recent discussion. In this section we introduce our notation, review the derivation of the UV fixed point, and calculate the anomalous dimensions of $\phi$ and $\phi^{2}$.

The model (2.1) can equivalently be expressed in terms of the auxiliary fields $\sigma$ and $\rho$, with the action,

$$
S=\int d^{3} x\left(\frac{1}{2}(\partial \phi)^{2}+\frac{1}{\sqrt{N}} \sigma \phi^{2}+\frac{g_{6}}{6 \sqrt{N}} \rho^{3}-\sigma \rho\right) .
$$

\footnotetext{
${ }^{2}$ Another class of models which have been of interest recently are the SYK/tensor models [19-25].
} 


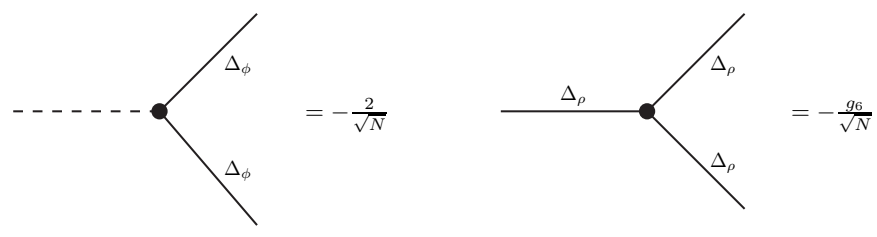

Figure 1. Feynman rules for the cubic interactions in (2.2).

The original action (2.1) is recovered from (2.2) by first integrating over the field $\sigma$ along the purely imaginary direction, which results in the delta-functional $\delta\left(\rho-\phi^{2} / \sqrt{N}\right)$, and then integrating over $\rho$. Note that the fields $\rho$ and $\phi$ are the only dynamical degrees of freedom in the large $N$ limit, because the propagator of $\sigma$ is suppressed by $1 / N$.

The propagator of the field $\phi$ in position space is,

$$
\langle\phi(x) \phi(0)\rangle=\frac{C_{\phi}}{|x|^{2 \Delta_{\phi}}},
$$

where the large $N$ amplitude and scaling dimension are given by,

$$
C_{\phi}=\frac{1}{4 \pi}, \quad \Delta_{\phi}=\frac{1}{2} .
$$

The corresponding Feynman rule for the normalized field is, ${ }^{3}$

$$
\stackrel{2 \Delta_{\phi} \quad \longrightarrow}{\bullet-\frac{1}{|x|^{2 \Delta_{\phi}}}}
$$

Notice that the quadratic part of the action (2.2) is not diagonal, which leads to a nontrivial merging relation between the fields $\rho$ and $\sigma$,

$$
\left\langle\rho\left(x_{1}\right) \sigma\left(x_{2}\right)\right\rangle=-\delta^{(3)}\left(x_{12}\right)=\bullet--\cdots
$$

Here the non-dynamical field $\sigma$ is denoted by a dashed line.

The Feynman rules for the cubic interactions in (2.2), $\phi^{2} \sigma$ and $\rho^{3}$, are shown in figure 1. They generate kinetic terms for $\sigma$ and $\rho$, respectively. However, the $\mathcal{O}\left(\rho^{2}\right)$ term in the effective action is suppressed by $1 / N$ relative to the $\mathcal{O}\left(\sigma^{2}\right)$. Indeed, the $1 / N$ factor from the cubic vertices is cancelled by $N$ fields $\phi$ running in the loop coming from $\sigma \phi^{2}$,

$$
-S_{\text {eff }} \supset C_{\phi}^{2} \int d^{3} x_{1} \int d^{3} x_{2} \frac{\sigma\left(x_{1}\right) \sigma\left(x_{2}\right)}{x_{12}^{2}}=\sigma\left(x_{1}\right)
$$

Inverting the quadratic part of the effective action yields,

$$
\langle\rho(x) \rho(0)\rangle=\frac{C_{\rho}}{|x|^{2 \Delta_{\rho}}},
$$

\footnotetext{
${ }^{3}$ In our conventions, all propagators in the Feynman diagrams are normalized to unity, and therefore each Feynman graph should be multiplied by the corresponding amplitudes.
} 
where the large $N$ amplitude and scaling dimension of the field $\rho$ are given by,

$$
C_{\rho}=2 C_{\phi}^{2}=\frac{1}{8 \pi^{2}}, \quad \Delta_{\rho}=1 .
$$

The corresponding Feynman rule for the normalized field is,

$$
\stackrel{2 \Delta_{\rho}}{\bullet}=\frac{1}{|x|^{2 \Delta_{\rho}}} .
$$

In the next subsection, we are going to use the background field method to calculate the effective cubic vertex in the effective action, at next-to-leading order in the $1 / N$ expansion. In preparation for this, here we establish an additional Feynman rule in the presence of a background field $\bar{\rho}(x)$, i.e. we substitute $\rho \rightarrow \rho+\bar{\rho}$ into (2.2) to get an action in the presence of $\bar{\rho}(x)$. The relevant Feynman rule is based on the observation that the linear coupling $\rho \sigma$ becomes $(\rho+\bar{\rho}) \sigma$. Hence, $\bar{\rho}$ is a source field for $\sigma$. In particular, it induces a background field $\bar{\sigma}(x)$, which can be derived by carrying out the gaussian integral over $\phi$ and eliminating the term $\bar{\rho} \sigma$ by a field redefinition $\sigma \rightarrow \sigma+\bar{\sigma}$, where

$$
\bar{\sigma}(x)=\frac{4}{\pi^{2}} \int d^{3} x_{1} \frac{\bar{\rho}\left(x_{1}\right)}{\left|x-x_{1}\right|^{4}}=\frac{4}{\pi^{2}} \times \stackrel{\bar{\rho}\left(x_{1}\right)}{{ }^{\longrightarrow}} \stackrel{x}{x}^{x}
$$

A quick consistency check can be carried out by contracting both sides of this expression with $\bar{\rho}\left(x_{2}\right)$, and using $(2.5),(2.6),(2.7)$, and the inverse propagator relation $(d=3, \Delta=2)$,

$$
\frac{1}{\pi^{d}} \frac{\Gamma(d-\Delta) \Gamma(\Delta)}{\Gamma\left(\frac{d}{2}-\Delta\right) \Gamma\left(\Delta-\frac{d}{2}\right)} \int d^{d} x_{2} \frac{1}{\left|x_{12}\right|^{2 \Delta}\left|x_{23}\right|^{2(d-\Delta)}}=\delta^{(d)}\left(x_{1}-x_{3}\right) .
$$

There are simple diagrammatical rules for performing some of the integrals involved in the conformal perturbation theory calculations we will encounter. For instance, a simple loop diagram satisfies additivity (in position space),

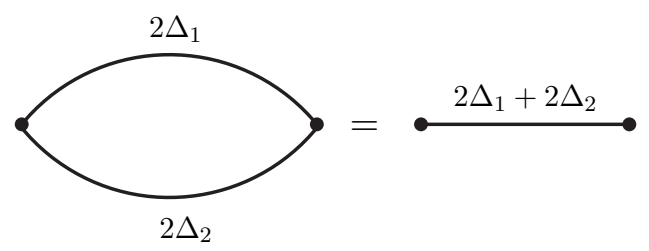

In addition, there is a propagator merging relation of the form,

$$
\int d^{d} x_{2} \frac{1}{\left|x_{12}\right|^{2 \Delta_{1}}\left|x_{23}\right|^{2 \Delta_{2}}}=\frac{U\left(\Delta_{1}, \Delta_{2}, d-\Delta_{1}-\Delta_{2}\right)}{\left|x_{13}\right|^{2\left(\Delta_{1}+\Delta_{2}\right)-d}}
$$

where

$$
U\left(\Delta_{1}, \Delta_{2}, \Delta_{3}\right)=\pi^{\frac{d}{2}} \frac{\Gamma\left(\frac{d}{2}-\Delta_{1}\right) \Gamma\left(\frac{d}{2}-\Delta_{2}\right) \Gamma\left(\frac{d}{2}-\Delta_{3}\right)}{\Gamma\left(\Delta_{1}\right) \Gamma\left(\Delta_{2}\right) \Gamma\left(\Delta_{3}\right)} .
$$

This relation can be represented diagrammatically as, ${ }^{4}$

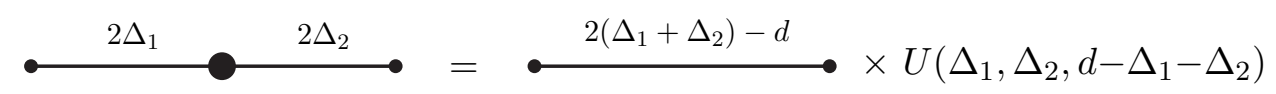

\footnotetext{
${ }^{4}$ The middle vertex on the left-hand side is integrated over.
} 


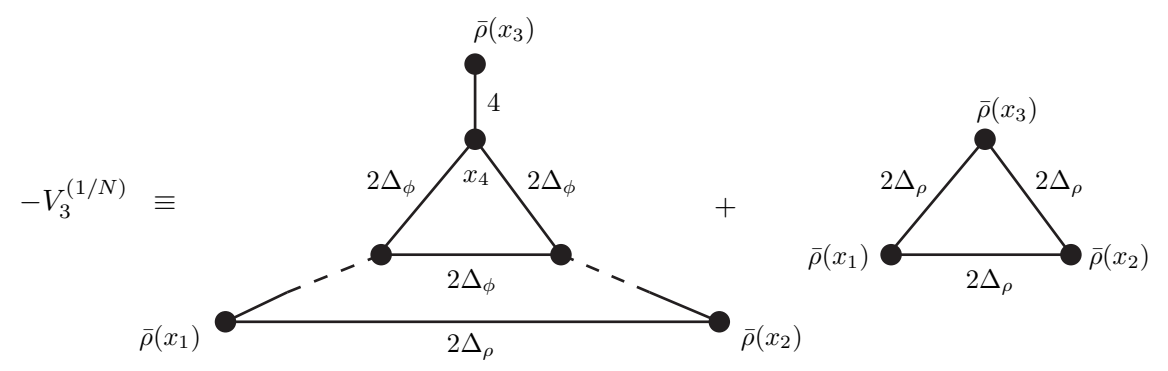

Figure 2. Subleading correction to the cubic vertex of the effective action. $\bar{\rho}(x)$ is a fixed background field.

Finally, the star-triangle relation for the cubic scalar vertex is given by,

$$
\begin{aligned}
\int d^{d} x_{4} \frac{1}{\left|x_{14}\right|^{2 \Delta_{1}}\left|x_{24}\right|^{2 \Delta_{2}}\left|x_{34}\right|^{2 \Delta_{3}}} & =\frac{U\left(\Delta_{1}, \Delta_{2}, \Delta_{3}\right)}{\left|x_{12}\right|^{d-2 \Delta_{3}}\left|x_{13}\right|^{d-2 \Delta_{2}}\left|x_{23}\right|^{d-2 \Delta_{1}}}, \\
\Delta_{1}+\Delta_{2}+\Delta_{3} & =d .
\end{aligned}
$$

Diagrammatically it takes the form,

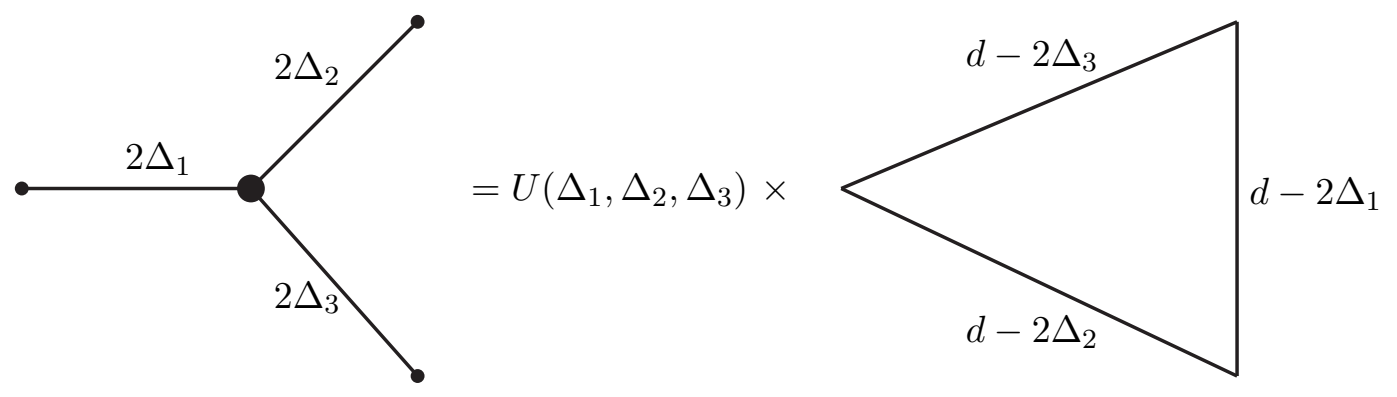

\subsection{UV fixed point}

The beta function of $g_{6}$ vanishes in the large $N$ limit. However, the two diagrams shown in figure 2 induce a non-trivial $\mathrm{RG}$ flow at the next-to-leading order. These diagrams represent a $1 / N$ correction to the cubic vertex of the effective action in the presence of the background field $\bar{\rho}(x),{ }^{5}$

$$
\begin{aligned}
V_{3}^{(1 / N)}= & \frac{16 C_{\phi}^{3} C_{\rho}}{\pi^{2} \sqrt{N}}\left(\frac{g_{6}}{\sqrt{N}}\right)^{2} \int \prod_{i=1}^{3} d^{3} x_{i} \int d^{3} x_{4} \frac{\bar{\rho}\left(x_{1}\right) \bar{\rho}\left(x_{2}\right) \bar{\rho}\left(x_{3}\right)}{\left|x_{12}\right|^{3}\left|x_{14}\right|\left|x_{24}\right|\left|x_{34}\right|^{4}} \\
& +\frac{C_{\rho}^{3}}{6}\left(\frac{g_{6}}{\sqrt{N}}\right)^{3} \int \prod_{i=1}^{3} d^{3} x_{i} \frac{\bar{\rho}\left(x_{1}\right) \bar{\rho}\left(x_{2}\right) \bar{\rho}\left(x_{3}\right)}{\left|x_{12}\right|^{2}\left|x_{13}\right|^{2}\left|x_{32}\right|^{2}}
\end{aligned}
$$

${ }^{5}$ When calculating the first diagram in figure 2, we used expression (2.8) for the induced background field $\bar{\sigma}(x)$. The latter is coupled to $\phi^{2}$ via a cubic vertex with amplitude $-2 / \sqrt{N}$. When writing down the contributions of these diagrams to $V_{3}^{(1 / N)}$, we accounted for the symmetry factors $1 / 2$ ! and $1 / 3$ !, respectively. 
The integral over $x_{4}$ in the first line can be carried out through the use of the star-triangle relation (2.12). ${ }^{6}$ We get,

$$
V_{3}^{(1 / N)}=\frac{-g_{6}^{2}}{16 \pi^{6} N^{3 / 2}}\left(1-\frac{g_{6}}{192}\right) \int \prod_{i=1}^{3} d^{3} x_{i} \frac{\bar{\rho}\left(x_{1}\right) \bar{\rho}\left(x_{2}\right) \bar{\rho}\left(x_{3}\right)}{\left|x_{12}\right|^{2}\left|x_{13}\right|^{2}\left|x_{23}\right|^{2}} .
$$

Naively, this vertex is conformally invariant, regardless of the value of $g_{6}$. However, this is deceptive, since it exhibits a logarithmic UV divergence when the three fields $\bar{\rho}(x)$ collide. This results in a non-trivial RG flow for $g_{6}$. To derive the associated beta function, it is sufficient to set all the fields to be at the same point $x_{1}$. Using the propagator merging relation (2.10) to integrate over $x_{2}$, and introducing both a UV cut-off $\mu_{0}$ and an IR cut-off $\mu$ to regularize the integral over $x_{3}$ yields, ${ }^{7}$

$$
V_{3}^{(1 / N)}=-\frac{g_{6}^{2}\left(\mu_{0}\right)}{4 \pi^{2} N^{3 / 2}}\left(1-\frac{g_{6}\left(\mu_{0}\right)}{192}\right) \log \left(\mu_{0} / \mu\right) \int d^{3} x_{1} \bar{\rho}^{3}\left(x_{1}\right)+\ldots
$$

where ellipsis encode finite terms.

Combining this result with the leading order cubic vertex, we deduce the following RG flow equation for the coupling,

$$
g_{6}(\mu)=g_{6}\left(\mu_{0}\right)-\frac{3 g_{6}^{2}\left(\mu_{0}\right)}{2 \pi^{2} N}\left(1-\frac{g_{6}\left(\mu_{0}\right)}{192}\right) \log \left(\mu_{0} / \mu\right)
$$

Equivalently, the beta function is,

$$
\beta\left(g_{6}\right)=\mu \frac{d g_{6}}{d \mu}=\frac{3 g_{6}^{2}}{2 \pi^{2} N}\left(1-\frac{g_{6}}{192}\right)+\mathcal{O}\left(1 / N^{2}\right) .
$$

In particular, the model exhibits a UV stable fixed point at $g_{6}^{*}=192$, as originally found in [26]. Note also that the $1 / N$ correction, $V_{3}^{(1 / N)}$, to the cubic vertex vanishes at the UV fixed point. We will use this fact later in section 3.2, when we derive the conformal three-point function $\langle\rho \rho \rho\rangle$.

\subsection{Anomalous dimensions of $\phi$ and $\phi^{2}$}

In this section we apply the background field method [29] to derive the anomalous dimension of $\phi$ and of $\rho \sim \phi^{2}{ }^{8}$ These two operators are special because, unlike composite operators

\footnotetext{
${ }^{6}$ The integral actually suffers from a power law divergence. However, such divergences depend on the choice of regularization scheme. For instance, they are absent within dimensional regularization. Therefore, we ignore them in what follows. Indeed, a similar remark applies to the integral identities, such as the propagator merging relation and the star-triangle relation. On the other hand, in general these relations are singular if the integral has a logarithmic divergence.

${ }^{7}$ We use UV/IR terminology in the Wilsonian sense. More specifically, the coupling $g_{6}$ of the original action (2.2) is defined at a certain (UV) scale $\mu_{0}$. It is denoted by $g_{6}\left(\mu_{0}\right)$ in (2.15) and (2.16). We are interested in finding the value of $g_{6}(\mu)$ at a lower (IR) scale $\mu$. If the theory is conformal, it looks the same at all scales, and there is no difference between the couplings. Unless $g_{6}=192$, this is not the case in the $\phi^{6}$ model.

Indeed, the $\phi^{6} \sim \rho^{3}$ interaction in (2.2) accumulates correction (2.15) at a lower (IR) scale $\mu$. This correction vanishes when $\mu=\mu_{0}$. Setting $\bar{\rho}$ to a constant value (zero mode) in (2.14) and evaluating the integrals over a shell $\mu_{0}^{-1} \lesssim|x| \lesssim \mu^{-1}$ is an alternative way of deriving (2.15). This is enough to get the RG flow of $g_{6}$, because the sextic interaction contributes to the effective potential.

${ }^{8}$ The identification $\rho \sim \phi^{2}$ follows from the equation of motion operator, i.e., vary (2.2) with respect to $\sigma$.
} 


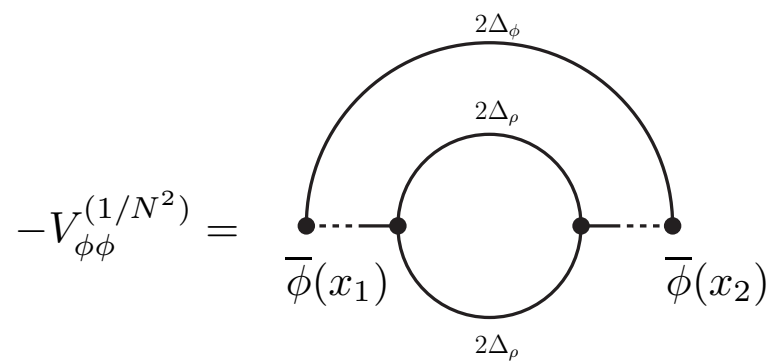

Figure 3. The $\mathcal{O}\left(1 / N^{2}\right)$ correction to the quadratic effective action of $\phi$, where $\bar{\phi}(x)$ is a given background field.

with higher powers of $\phi$, their anomalous dimensions vanish at leading and next-to-leading order in $1 / N$. One has to consider $\mathcal{O}\left(1 / N^{2}\right)$ diagrams to find the first non-trivial correction.

For instance, the anomalous dimension of $\phi$ (denoted by $\gamma_{\phi}$ ) is entirely fixed by the diagram in figure 3. It represents the leading order correction to the quadratic effective action of the scalar field. Using the Feynman rules gives,

$$
-V_{\phi \phi}^{\left(1 / N^{2}\right)}=\frac{1}{4}\left(\frac{g_{6}}{\sqrt{N}}\right)^{2}\left(\frac{2}{\sqrt{N}}\right)^{2} C_{\phi} C_{\rho}^{2} \int d^{3} x_{1} \int d^{3} x_{2} \frac{\bar{\phi}\left(x_{1}\right) \bar{\phi}\left(x_{2}\right)}{\left|x_{12}\right|^{5}} .
$$

This term is singular in the vicinity of $x_{1} \sim x_{2}$. The logarithmic divergence is associated with the anomalous dimension of $\phi$, and we isolate it by expanding the background field $\bar{\phi}\left(x_{2}\right)$ around $x_{1}$,

$$
V_{\phi \phi}^{\left(1 / N^{2}\right)}=-\frac{g_{6}^{2}}{2 N^{2}} C_{\phi} C_{\rho}^{2} \int d^{3} x_{1} \bar{\phi}\left(x_{1}\right) \partial_{\mu} \partial_{\nu} \bar{\phi}\left(x_{1}\right) \int d^{3} x_{2} \frac{x_{12}^{\mu} x_{12}^{\nu}}{\left|x_{12}\right|^{5}}+\ldots
$$

where ellipsis encode terms which do not contribute to $\gamma_{\phi}$. Introducing a spherical sharp cut-off $\mu$, and substituting the fixed point value $g_{6}^{*}=192$, yields

$$
V_{\phi \phi}^{\left(1 / N^{2}\right)}=\frac{96}{\pi^{4} N^{2}} \int d^{3} x \partial_{\mu} \bar{\phi} \partial^{\mu} \bar{\phi} \log (\mu|x|)+\ldots
$$

The value of $\gamma_{\phi}$ can be obtained by comparing this expression with the full propagator of $\phi$,

$$
\langle\phi(x) \phi(0)\rangle=\mu^{-2 \gamma_{\phi}} \frac{C_{\phi}\left(1+A_{\phi}\right)}{|x|^{1+2 \gamma_{\phi}}},
$$

where $A_{\phi}$ is associated with higher order corrections to the leading order amplitude $C_{\phi}$. Hence,

$$
\gamma_{\phi}=\frac{96}{\pi^{4} N^{2}}+\mathcal{O}\left(1 / N^{3}\right) .
$$

in full agreement with [26].

Next we consider the field $\rho$. Its full propagator can be written as,

$$
\langle\rho(x) \rho(0)\rangle=\mu^{-2 \gamma_{\rho}} \frac{C_{\rho}\left(1+A_{\rho}\right)}{|x|^{2\left(1+\gamma_{\rho}\right)}},
$$

where $\gamma_{\rho}$ is the anomalous dimension, and $A_{\rho}$ is associated with $1 / N$ corrections to the leading order amplitude $C_{\rho}$. 


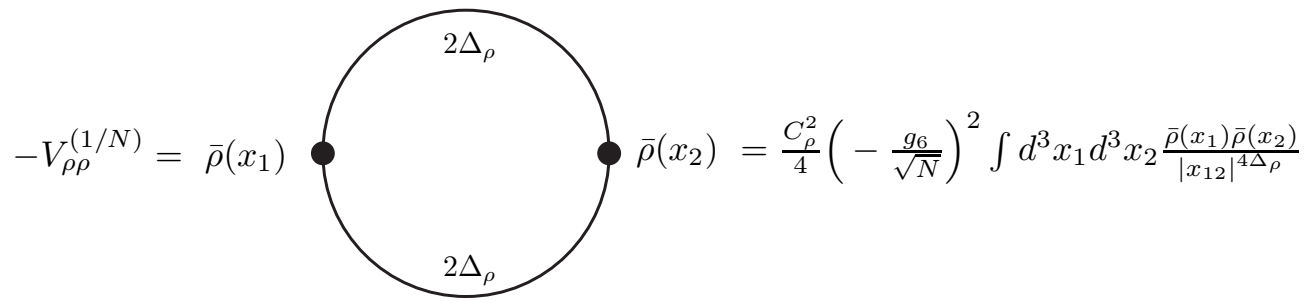

Figure 4. The $\mathcal{O}(1 / N)$ correction to the quadratic effective action of $\rho$, where $\bar{\rho}$ is the background field. The solid dots represent the cubic vertex with one of the legs substituted by the background $\bar{\rho}$, i.e. $-\frac{g_{6}}{\sqrt{N}} \bar{\rho}$, and $1 / 4$ is the symmetry factor.

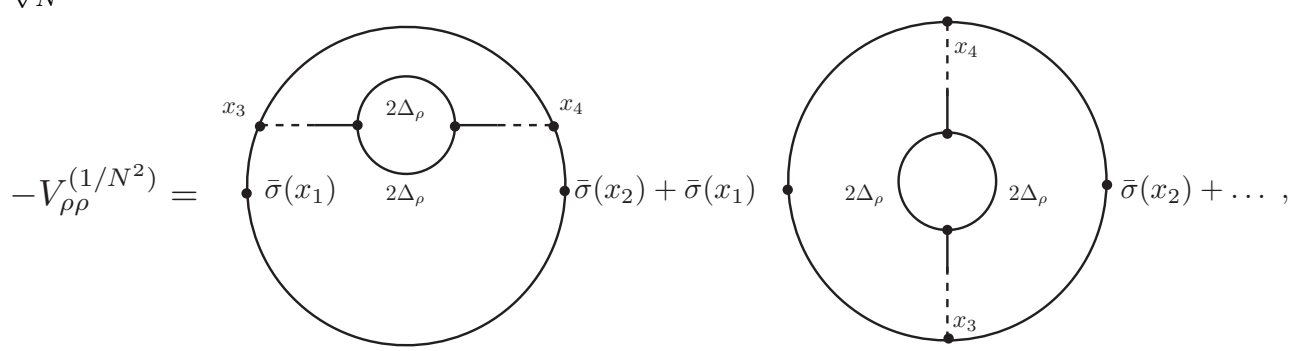

Figure 5. The $\mathcal{O}\left(1 / N^{2}\right)$ correction to the quadratic effective action of $\rho$. The background field is $\bar{\sigma} \sim \bar{\rho}$, given by (2.8). All propagators on the outer circles of these diagrams correspond to the field $\phi$ (the label $2 \Delta_{\phi}$ on the lines is suppressed). Ellipsis represent $\mathcal{O}\left(1 / N^{2}\right)$ graphs which do not contribute to the anomalous dimension $\gamma_{\rho}$.

The two-point effective vertex at order $1 / N$ in the presence of the background field $\bar{\rho}(x)$ is shown in figure 4 . It is finite and contributes to $A_{\rho}$ only; we will calculate it in the next section. Here our aim is to evaluate the anomalous dimension $\gamma_{\rho}$. We therefore proceed to the $\mathcal{O}\left(1 / N^{2}\right)$ diagrams which contribute to the effective two-point vertex. They can be obtained, for example, by dressing the vertices and propagators in figure 4 . However, such diagrams are either finite or vanish, because the $\rho$ propagator has no divergences at order $1 / N$, whereas, as we argued in the previous subsection, the next-to-leading order correction to the cubic vertex vanishes at the fixed point. Hence, the only divergent diagrams which contribute to $\gamma_{\rho}$ are shown in figure 5 .

Using the Feynman rules yields,

$$
\begin{aligned}
-V_{\rho \rho}^{\left(1 / N^{2}\right)}= & \frac{N}{4}\left(\frac{-g_{6}}{\sqrt{N}}\right)^{2}\left(\frac{2}{\sqrt{N}}\right)^{4} C_{\phi}^{4} C_{\rho}^{2}\left(\int \prod_{i=1}^{4} d^{3} x_{i} \frac{\bar{\sigma}\left(x_{1}\right) \bar{\sigma}\left(x_{2}\right)}{\left|x_{12}\right|\left|x_{13}\right|\left|x_{34}\right|^{5}\left|x_{24}\right|}\right. \\
& \left.+\frac{1}{2} \int \prod_{i=1}^{4} d^{3} x_{i} \frac{\bar{\sigma}\left(x_{1}\right) \bar{\sigma}\left(x_{2}\right)}{\left|x_{13}\right|\left|x_{23}\right|\left|x_{14}\right|\left|x_{24}\right|\left|x_{34}\right|^{4}}\right)+\ldots
\end{aligned}
$$

The integral over $x_{4}$ in the first expression within the parenthesis is power law divergent in the vicinity of $x_{4} \approx x_{3}$. In principle, it could have a logarithmic divergence, but its coefficient vanishes; this can be seen by introducing a spherical sharp cut-off $\mu$, and expanding $\left|x_{4}-x_{2}\right|^{-1}$ around $x_{4}=x_{3}$.

The power law divergent terms do not contribute to $\gamma_{\rho}$, while the finite part of the integral over $x_{4}$ can be evaluated by analytic continuation in the scaling dimension of 
the field $\rho$, or by dimensional regularization. In both regularization schemes, one can use the propagator merging relation and remove the regulator at the end. This procedure is sufficient to recover the contribution to $\gamma_{\rho}$. The result is,

$$
\int d^{3} x_{4} \frac{1}{\left|x_{34}\right|^{5}\left|x_{42}\right|}=-\frac{2 \pi}{3} \frac{1}{\left|x_{32}\right|^{3}} .
$$

Similarly, the integral over $x_{4}$ in the second expression within the parenthesis in (2.24) can be calculated by using the star-triangle relation (2.12),

$$
\int d^{3} x_{4} \frac{1}{\left|x_{14}\right|\left|x_{24}\right|\left|x_{34}\right|^{4}}=\frac{-2 \pi\left|x_{12}\right|}{\left|x_{13}\right|^{2}\left|x_{23}\right|^{2}}
$$

Hence,

$$
V_{\rho \rho}^{\left(1 / N^{2}\right)}=\frac{6}{N^{2} \pi^{7}}\left(\int \prod_{i=1}^{3} d^{3} x_{i} \frac{\bar{\sigma}\left(x_{1}\right) \bar{\sigma}\left(x_{2}\right)}{\left|x_{12}\right|\left|x_{13}\right|\left|x_{23}\right|^{3}}+\frac{3}{2} \int \prod_{i=1}^{3} d^{3} x_{i} \frac{\bar{\sigma}\left(x_{1}\right) \bar{\sigma}\left(x_{2}\right)\left|x_{12}\right|}{\left|x_{13}\right|^{3}\left|x_{23}\right|^{3}}\right)+\ldots
$$

The remaining integrals over $x_{3}$ are logarithmically divergent. Using a spherical sharp cut-off $\mu$ yields,

$$
\int d^{3} x_{3} \frac{1}{\left|x_{13}\right|^{3}\left|x_{23}\right|^{3}}=\frac{8 \pi}{\left|x_{12}\right|^{3}} \log \left(\mu\left|x_{12}\right|\right)+\ldots
$$

Thus,

$$
V_{\rho \rho}^{\left(1 / N^{2}\right)}=\frac{96}{N^{2} \pi^{6}} \int \prod_{i=1}^{2} d^{3} x_{i} \frac{\bar{\sigma}\left(x_{1}\right) \bar{\sigma}\left(x_{2}\right)}{\left|x_{12}\right|^{2}} \log \left(\mu\left|x_{12}\right|\right)+\ldots .
$$

Or, equivalently, substituting (2.8) and using the inverse propagator relation (2.9), we obtain

$$
V_{\rho \rho}^{\left(1 / N^{2}\right)}=\frac{-3072}{N^{2} \pi^{6}} \int \prod_{i=1}^{2} d^{3} x_{i} \frac{\bar{\rho}\left(x_{1}\right) \bar{\rho}\left(x_{2}\right)}{\left|x_{12}\right|^{4}} \log \left(\mu\left|x_{12}\right|\right)+\ldots,
$$

Comparing to (2.23), we get the anomalous dimension,

$$
\gamma_{\rho}=\frac{768}{N^{2} \pi^{4}}+\mathcal{O}\left(1 / N^{3}\right)
$$

This result does not match $[26]$.

\section{CFT data for the critical $\phi^{6}$ model}

In this section we use large $N$ techniques to derive the OPE coefficients and anomalous dimensions of various primary operators in the critical $\phi^{6}$ model. Our calculations extend known results in the literature [26].

\subsection{Anomalous dimensions of $\left(\phi^{2}\right)^{n}$}

In the large $N$ limit there are two dynamical fields, $\phi$ and $\rho=\phi^{2} / \sqrt{N}$, with scaling dimensions $\Delta_{\phi}=1 / 2$ and $\Delta_{\rho}=1$, respectively. Their propagators are given by $(2.3)$ and (2.6). In contrast, the dynamics of the field $\sigma \sim \rho^{2} / \sqrt{N}$ is suppressed by $1 / N$. 
As we saw in the previous section, higher order corrections induce anomalous dimensions to the leading order scalings of the fields. In this subsection, we calculate the anomalous dimension of the composite operators $\left(\phi^{2}\right)^{n}$. The subleading terms in the propagator, both the anomalous dimension and the amplitude corrections, also contribute to various next-to-leading order three-point functions, and will be needed for the computation of the OPE coefficients, to be performed in the next subsection.

The full propagator of the field $\rho$ is given by (2.23). The amplitude correction $A_{\rho}$ at the next-to-leading order is entirely fixed by attaching two leading-order propagators (2.6) to the one-loop diagram shown earlier in figure 4. As a result, the $\mathcal{O}(1 / N)$ correction to $(2.6)$ is given by,

$$
\delta\left\langle\rho\left(x_{3}\right) \rho(0)\right\rangle=\frac{1}{2}\left(\frac{-g_{6}}{\sqrt{N}}\right)^{2} C_{\rho}^{4} \int \frac{d^{3} x_{1} d^{3} x_{2}}{\left|x_{1}\right|^{2 \Delta_{\rho}}\left|x_{12}\right|^{4 \Delta_{\rho}}\left|x_{32}\right|^{2 \Delta_{\rho}}} .
$$

The integrals can be evaluated by using (2.9). At the critical point, $g_{6}^{*}=192$, we obtain,

$$
A_{\rho}=\frac{1}{2}\left(\frac{-g_{6}}{\sqrt{N}}\right)^{2} C_{\rho}^{3}\left(-2 \pi^{4}\right)=-\frac{g_{6}^{2}}{512 \pi^{2} N}=-\frac{72}{\pi^{2} N}, \quad \gamma_{\rho}=0+\mathcal{O}\left(1 / N^{2}\right) .
$$

While the anomalous dimension of $\rho$ is highly suppressed, this is not the case for $\rho^{n} \sim\left(\phi^{2}\right)^{n}$ with $n \geq 2$. To leading order in $1 / N$ we have

$$
\left\langle\rho(x)^{n} \rho(0)^{n}\right\rangle=n ! \frac{C_{\rho}^{n}}{|x|^{2 n}} .
$$

Or, diagrammatically,

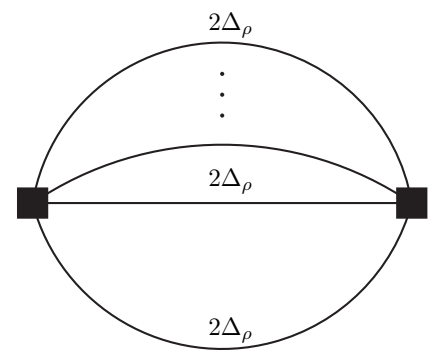

where the black squares stand for insertions of $\rho^{n}$, and dots represent additional lines (since $n$ is general). The full propagator, which includes subleading corrections to (3.3), will take the form,

$$
\left\langle\rho(x)^{n} \rho(0)^{n}\right\rangle=n ! \mu^{-2 \gamma_{n}} \frac{C_{\rho}^{n}\left(1+A_{n}\right)}{|x|^{2\left(n+\gamma_{n}\right)}},
$$

where $A_{n}$ and $\gamma_{n}$ are the $1 / N$ corrections to the amplitude and the anomalous dimension, respectively.

There are four diagrams which contribute to the full propagator at order $1 / N$. Two of them are shown in figure 6 . They are finite and contribute only to $A_{n}$,

$$
\begin{aligned}
C_{1} & =\frac{n n !}{2}\left(\frac{-g_{6}}{\sqrt{N}}\right)^{2} \frac{C_{\rho}^{n+3}}{\left|x_{3}\right|^{2(n-1)}} \int \frac{d^{3} x_{1} d^{3} x_{2}}{\left|x_{1}\right|^{2}\left|x_{12}\right|^{4}\left|x_{32}\right|^{2}}, \\
C_{2} & =\frac{n(n-1) n !}{2}\left(\frac{-g_{6}}{\sqrt{N}}\right)^{2} \frac{C_{\rho}^{n+3}}{\left|x_{3}\right|^{2(n-2)}} \int \frac{d^{3} x_{1} d^{3} x_{2}}{\left|x_{1}\right|^{4}\left|x_{12}\right|^{2}\left|x_{32}\right|^{4}} .
\end{aligned}
$$



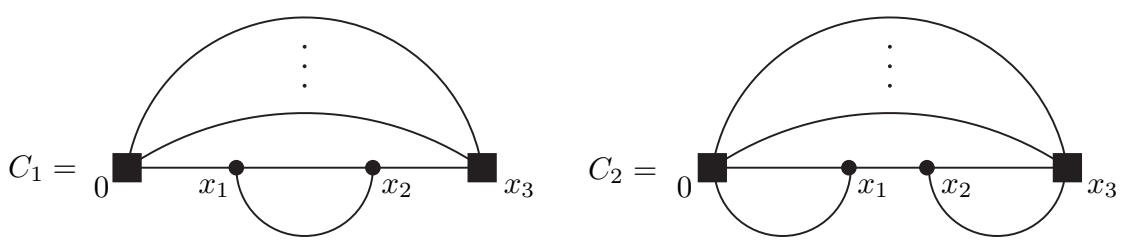

Figure 6. Finite diagrams which contribute to the full correlator $\left\langle\rho^{n}\left(x_{3}\right) \rho^{n}(0)\right\rangle$ at order $1 / N$. The black squares denote insertions of the composite operator $\rho^{n}$. All propagators are propagators of $\rho,(2.6)$.
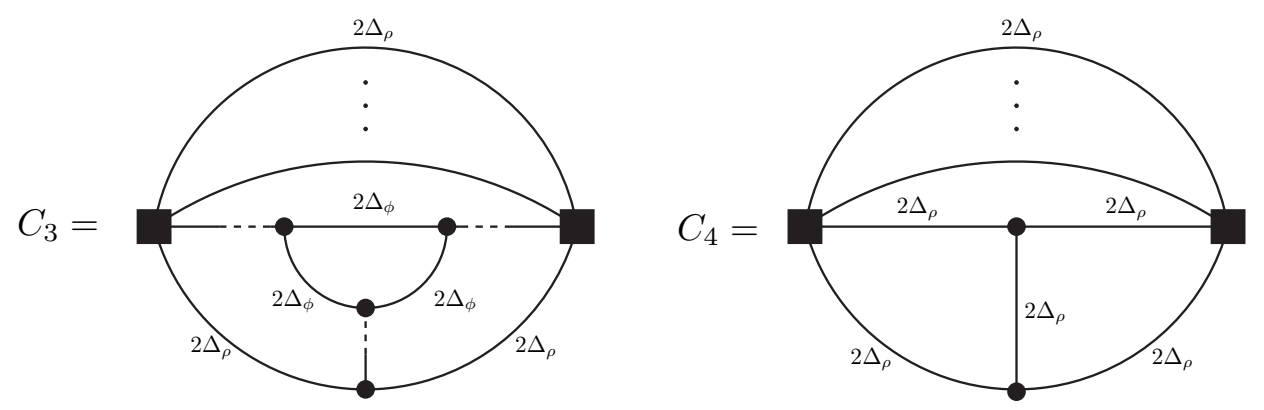

Figure 7. Divergent diagrams which contribute to the full correlator $\left\langle\rho^{n}\left(x_{3}\right) \rho^{n}(0)\right\rangle$ at order $1 / N$. The black squares denote insertions of the composite operator $\rho^{n}$.

The one-loop subdiagrams of $C_{1}$ and $C_{2}$ can be evaluated using (2.9), to get,

$$
C_{1}+C_{2}=n^{2} n ! \frac{C_{\rho}^{n}}{\left|x_{3}\right|^{2 n}} A_{\rho}
$$

where $A_{\rho}$ is given by (3.2).

In addition, there are two divergent diagrams shown in figure 7 . These diagrams are responsible for the anomalous dimension. Applying the Feynman rules gives,

$$
\begin{aligned}
C_{3} & =-n(n-1) n ! N\left(\frac{-2}{\sqrt{N}}\right)^{3}\left(\frac{-g_{6}}{\sqrt{N}}\right) \frac{C_{\rho}^{n} C_{\phi}^{3}}{\left|x_{3}\right|^{2 n-3}} \int \frac{d^{3} x_{2}}{\left|x_{2}\right|^{3}\left|x_{23}\right|^{3}}, \\
C_{4} & =\frac{n(n-1) n !}{2}\left(\frac{-g_{6}}{\sqrt{N}}\right)^{2} \frac{C_{\rho}^{n+3}}{\left|x_{3}\right|^{2(n-2)}} \int \frac{d^{3} x_{1} d^{3} x_{2}}{\left|x_{1}\right|^{2}\left|x_{2}\right|^{2}\left|x_{12}\right|^{2}\left|x_{13}\right|^{2}\left|x_{23}\right|^{2}} .
\end{aligned}
$$

These expressions can be combined after carrying out the integral over $x_{3}$, through the use of the star-triangle relation (2.12),

$$
C_{3}+C_{4}=-n(n-1) n ! \frac{8 g_{6}}{N}\left(1-\frac{g_{6}}{128}\right) \frac{C_{\rho}^{n} C_{\phi}^{3}}{\left|x_{3}\right|^{2 n-3}} \int \frac{d^{3} x_{2}}{\left|x_{2}\right|^{3}\left|x_{23}\right|^{3}} .
$$

The integral over $x_{2}$ diverges logarithmically. This divergence is associated with the anomalous dimension of $\rho^{n}$. Using (2.28) yields,

$$
C_{3}+C_{4}=-n(n-1) n ! \frac{g_{6}}{\pi^{2} N} C_{\rho}^{n}\left(1-\frac{g_{6}}{128}\right) \frac{1}{\left|x_{3}\right|^{2 n}} \log \left(\mu\left|x_{3}\right|\right)+\ldots
$$



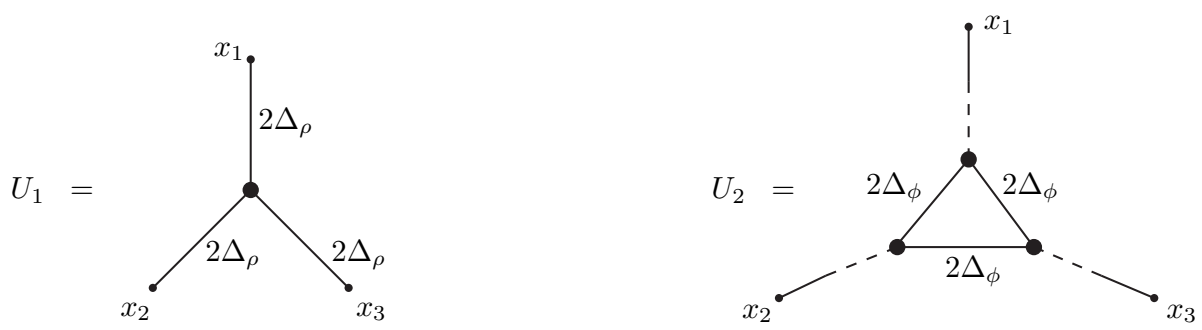

Figure 8. Leading order Feynman diagrams of the three-point function $\langle\rho \rho \rho\rangle$.

Combining (3.6) and (3.9) with the leading order propagator (3.3), and matching the result with the general form (3.4), gives

$$
\gamma_{n}=\frac{n(n-1) g_{6}}{2 \pi^{2} N}\left(1-\frac{g_{6}}{128}\right)+\mathcal{O}\left(1 / N^{2}\right)=-\frac{48 n(n-1)}{\pi^{2} N}+\mathcal{O}\left(1 / N^{2}\right) .
$$

The anomalous dimensions for $n=1,2$ match $[26],{ }^{9}$ while the anomalous dimensions $\gamma_{n}$ for $n \geq 3$ are new. As an additional check of our result, we note that the anomalous dimension of $\phi^{6}$ indeed satisfies $\gamma_{3}=\frac{\partial \beta}{\partial g_{6}}=-\frac{288}{\pi^{2} N}$.

\subsection{OPE coefficients}

In this section we derive several OPE coefficients, up to the next-to-leading order in $1 / N$.

Evaluation of $\left\langle\phi^{2} \phi^{2} \phi^{2}\right\rangle$. There are two Feynman diagrams which contribute to the leading order three-point function $\langle\rho \rho \rho\rangle$, as shown in figure 8. Using the star-triangle relation (2.12) to integrate over the cubic vertex in $U_{1}$ yields,

$$
U_{1}=-\frac{g_{6} C_{\rho}^{3}}{\sqrt{N}} \int d^{3} x_{4} \frac{1}{\left(\left|x_{14}\right|\left|x_{24}\right|\left|x_{34}\right|\right)^{2}}=-\frac{g_{6}}{(8 \pi)^{3} \sqrt{N}} \frac{1}{\left|x_{12}\right|\left|x_{13}\right|\left|x_{23}\right|} .
$$

The other diagram, $U_{2}$, is trivial to compute, as all three integrals are taken over delta functions (2.5), giving,

$$
U_{2}=\frac{8 C_{\phi}^{3}}{\sqrt{N}} \frac{1}{\left|x_{12}\right|\left|x_{13}\right|\left|x_{23}\right|}=\frac{1}{8 \pi^{3} \sqrt{N}} \frac{1}{\left|x_{12}\right|\left|x_{13}\right|\left|x_{23}\right|} .
$$

Thus, at the fixed point $g_{6}^{*}=192$, we get,

$$
\left\langle\rho\left(x_{1}\right) \rho\left(x_{2}\right) \rho\left(x_{3}\right)\right\rangle=U_{1}+U_{2}=\frac{C_{\rho \rho \rho}}{\left|x_{12}\right|\left|x_{13}\right|\left|x_{23}\right|}, \quad C_{\rho \rho \rho}=-\frac{1}{4 \pi^{3} \sqrt{N}}(1+\mathcal{O}(1 / N)) .
$$

It is standard to define the OPE coefficients to be for the fields which are normalized such that the amplitude of the two-point correlation function is unity. Based on (2.23), we therefore rescale the field $\rho$,

$$
\rho \rightarrow \sqrt{C_{\rho}\left(1+A_{\rho}\right)} \rho
$$

\footnotetext{
${ }^{9}$ In fact, $\gamma_{2}$ in [26] has the opposite sign.
} 


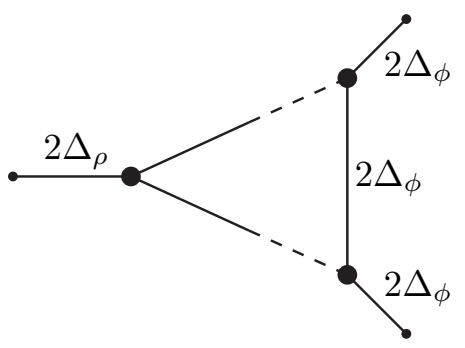

Figure 9. The $\mathcal{O}(1 / N)$ correction to the three-point function $\langle\phi \phi \rho\rangle$.

and get,

$$
\left.\left\langle\rho\left(x_{1}\right) \rho\left(x_{2}\right) \rho\left(x_{3}\right)\right\rangle\right|_{\text {normalized }}=\frac{\hat{C}_{\rho \rho \rho}}{\left|x_{12}\right|\left|x_{13}\right|\left|x_{23}\right|},
$$

where the OPE coefficient is given by

$$
\hat{C}_{\rho \rho \rho}=-4 \sqrt{\frac{2}{N}}(1+\mathcal{O}(1 / N))
$$

The next-to-leading order correction to this OPE coefficient follows directly from our previous results. In particular, it is entirely determined by the $1 / N$ corrections to the external propagators and to the cubic vertex. The former is encoded in the coefficient $A_{\rho}$ given by (3.2), whereas the latter, as argued in section 2.1 , vanishes. Hence, for $\rho$ normalized according to (3.14), we find that OPE coefficient up to order $1 / N$ is,

$$
\hat{C}_{\rho \rho \rho}=-4 \sqrt{\frac{2}{N}}\left(1+\frac{3}{2} A_{\rho}+\mathcal{O}\left(1 / N^{2}\right)\right) .
$$

Evaluation of $\left\langle\phi \phi \phi^{2}\right\rangle$. We now consider the three-point function $\left\langle\phi \phi \phi^{2}\right\rangle \sim\langle\phi \phi \rho\rangle$. To leading order in $1 / N$ it is determined by a tree diagram of the form

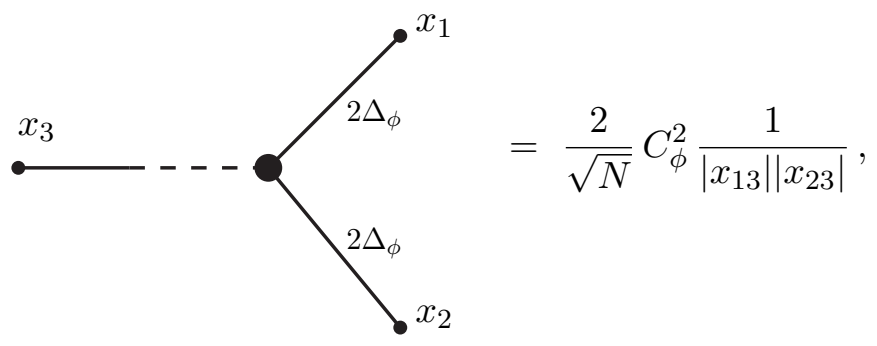

where we used the Feynman rules of section 2 to get the expression on the right hand side.

The next-to-leading order correction is obtained by accounting for the $1 / N$ corrections to the external propagators and the $\rho \phi^{2}$ vertex. The correction to the cubic vertex is represented by the one-loop diagram in figure 9 . It vanishes, because the integrals over the delta functions (2.5) result in $\left\langle\phi^{2}\right\rangle=0$.

Furthermore, as argued in section 3.1, only the amplitude of the $\rho$ propagator is modified at the next-to-leading order, whereas the anomalous dimension and the $1 / N$ correction to the $\phi$ propagator are absent at this order. As a result, the three-point function $\langle\phi \phi \rho\rangle$ up to order $1 / N$ is given by,

$$
\left\langle\phi\left(x_{1}\right) \phi\left(x_{2}\right) \rho\left(x_{3}\right)\right\rangle=\frac{2}{\sqrt{N}} \frac{C_{\phi}^{2}}{\left|x_{13}\right|\left|x_{23}\right|}\left(1+A_{\rho}+\mathcal{O}\left(1 / N^{2}\right)\right) .
$$


To get the OPE coefficient, we want to first properly normalize the fields: we rescale $\rho$, as was done in (3.14), and similarly rescale $\phi \rightarrow \sqrt{C_{\phi}\left(1+A_{\phi}\right)} \phi$, see (2.21). Since $A_{\phi} \sim \mathcal{O}\left(1 / N^{2}\right)$ and $A_{\rho}$ is given by (3.2), we get

$$
\left.\left\langle\phi\left(x_{1}\right) \phi\left(x_{2}\right) \rho\left(x_{3}\right)\right\rangle\right|_{\text {normalized }}=\frac{\hat{C}_{\phi \phi \rho}}{\left|x_{13}\right|\left|x_{23}\right|}, \quad \hat{C}_{\phi \phi \rho}=\sqrt{\frac{2}{N}}\left(1-\frac{36}{\pi^{2} N}+\mathcal{O}\left(1 / N^{2}\right)\right) .
$$

Notice that (3.19) is conformally covariant, because $2 \Delta_{\phi}-\Delta_{\rho}=0$ and, as argued in section $2.2, \gamma_{\phi} \sim \gamma_{\rho} \sim \mathcal{O}\left(1 / N^{2}\right)$.

\section{Review of the Gross-Neveu model}

In this section we review some aspects of the Gross-Neveu model [14] which will be needed for section 5 . Those who are familiar with the subject may proceed directly to the next section.

Consider the $U(n)$-invariant Gross-Neveu model in $d$-dimensional Euclidean space,

$$
\left.S=\int d^{d} x\left(\bar{\psi} \gamma^{\mu} \partial_{\mu} \psi+\frac{g}{N}(\bar{\psi} \psi)^{2}\right)\right)
$$

where $\psi$ collectively denotes $n$ Dirac fermions, and $\bar{\psi}=\psi^{\dagger}$ denotes the standard Hermitian conjugation. We choose conventions with Hermitian $\gamma$-matrices, $\left(\gamma^{\mu}\right)^{\dagger}=\gamma^{\mu}$, and, as is standard in large- $N$ fermionic models, take $N=n \operatorname{tr} \mathbb{I}$ where $\mathbb{I}$ is the identity matrix in the space of $2^{[d / 2]}$-dimensional Dirac spinors [15].

The standard trick for solving the Gross-Neveu model is to rewrite the model (4.1) using the Hubbard-Stratonovich field $s$,

$$
S=\int d^{d} x\left(\bar{\psi} \gamma^{\mu} \partial_{\mu} \psi-\frac{1}{4 g} s^{2}+\frac{1}{\sqrt{N}} s \bar{\psi} \psi\right)
$$

The coupling $g$ is irrelevant at the Gaussian fixed point, and the model is therefore IR free in dimensions $d>2$. However, the $\epsilon$-expansion suggests that it has a non-trivial UV fixed point, and the corresponding CFT has been extensively studied [15, 34-42]. In strictly two dimensions, the model (4.2) is a renowned example of an asymptotically free field theory [14]. ${ }^{10}$ As we will review in the next subsection, the large- $N$ scaling dimension of $s$ at the UV fixed point is equal to one, and so the corresponding CFT is unitary provided that $d \leq 4$.

\subsection{Feynman rules and useful identities}

In momentum space the bare propagators of the fields in (4.2) are given by, ${ }^{11}$

$$
\langle s(p) s(q)\rangle_{\mathrm{bare}}=(2 \pi)^{d} \delta^{(d)}(p+q)(-2 g), \quad\langle\psi(p) \bar{\psi}(q)\rangle=(2 \pi)^{d} \delta^{(d)}(p+q) \frac{-i p^{\mu} \gamma^{\mu}}{p^{2}},
$$

\footnotetext{
${ }^{10}$ See [43] and references therein for four-loop renormalization of the two-dimensional Gross-Neveu model.

${ }^{11}$ We will generally not keep track of the $U(n)$ indices.
} 

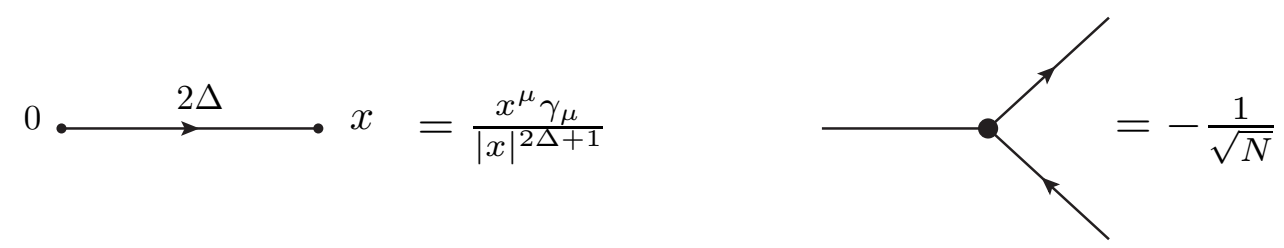

Figure 10. Feynman rules in the presence of Dirac field.

Since we are interested in the large- $N$ limit of the model, we have to resum the so-called bubble diagrams to get the leading order propagator of the auxiliary field $s$ [14],

$$
\langle s(p) s(q)\rangle_{N \rightarrow \infty}=-2 g \sum_{n=0}^{\infty}(-2 g B(p))^{n}=\frac{-2 g}{1+2 g B(p)} \quad \underset{\mathrm{UV}}{\rightarrow}-\frac{1}{B(p)} .
$$

In the last step we took the limit of large momentum, to ensure that the model is sitting at the UV fixed point. The fermionic bubble $B(p)$ appearing above is given by,

$$
B(p)=\frac{n}{N} \operatorname{tr} \int \frac{d^{d} q}{(2 \pi)^{d}} \frac{-i \gamma^{\mu} q_{\mu}(-i) \gamma^{\nu}(p-q)_{\nu}}{q^{2}(p-q)^{2}}=\frac{p^{d-2}}{4^{d-1} \pi^{\frac{d-3}{2}} \sin \left(\frac{\pi d}{2}\right) \Gamma\left(\frac{d-1}{2}\right)} .
$$

Using the standard Fourier transform relation,

$$
\int \frac{d^{d} x}{(2 \pi)^{d}} e^{i k \cdot x} \frac{1}{|k|^{d-2 \Delta}}=\frac{2^{2 \Delta-d}}{\pi^{d / 2}} \frac{1}{A(\Delta)} \frac{1}{|x|^{2 \Delta}}, \quad A(\Delta)=\frac{\Gamma\left(\frac{d}{2}-\Delta\right)}{\Gamma(\Delta)},
$$

we get the conformal propagator of the auxiliary field $s$ in position space [15] (see also $[41,42])$,

$$
\langle s(x) s(0)\rangle=\frac{C_{s}}{|x|^{2 \Delta_{s}}}, \quad C_{s}=-\frac{2^{d} \sin \left(\frac{\pi d}{2}\right) \Gamma\left(\frac{d-1}{2}\right)}{\pi^{\frac{3}{2}} \Gamma\left(\frac{d}{2}-1\right)}, \quad \Delta_{s}=1 .
$$

Similarly, the propagator of the fermion field $\psi$ is,

$$
\langle\psi(x) \bar{\psi}(0)\rangle=C_{\psi} \frac{x^{\mu} \gamma_{\mu}}{|x|^{2 \Delta_{\psi}+1}}, \quad C_{\psi}=\frac{\Gamma\left(\frac{d}{2}\right)}{2 \pi^{\frac{d}{2}}}, \quad \Delta_{\psi}=\frac{d-1}{2} .
$$

We will use position space Feynman rules, as we did in the previous sections for the scalar field theory. Since we now have fermions, a Dirac propagator will have an arrow, and there is no symmetry factor of 2 in the $\bar{\psi} \psi s$ vertex, see figure 10 . The amplitudes of the propagators are normalized to unity, and therefore one needs to multiply each Feynman diagram by an appropriate power of $C_{\psi, s}$.

The integral identities that were used in scalar conformal perturbation theory can be generalized to accommodate fermions [34] (see also [44] for a recent review), e.g.,
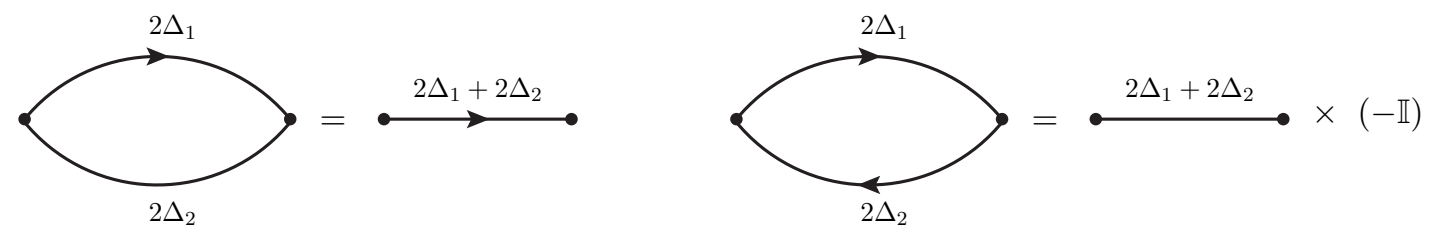
Moreover, the propagator merging relations with fermions take the form,

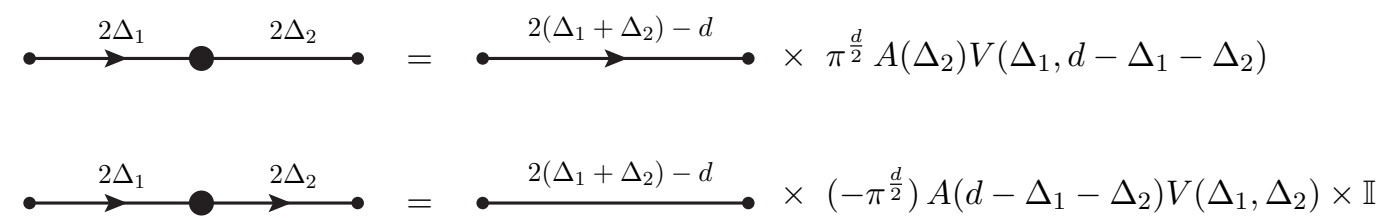

where the insertion point of the middle vertex on the left-hand side is integrated over, $A(\Delta)$ is defined in (4.6), and

$$
V\left(\Delta_{1}, \Delta_{2}\right)=\frac{\Gamma\left(\frac{d}{2}-\Delta_{1}+\frac{1}{2}\right)}{\Gamma\left(\Delta_{1}+\frac{1}{2}\right)} \frac{\Gamma\left(\frac{d}{2}-\Delta_{2}+\frac{1}{2}\right)}{\Gamma\left(\Delta_{2}+\frac{1}{2}\right)} .
$$

In the case of a Yukawa vertex, the star-triangle relation (2.12) becomes $\left(\Delta_{1}+\Delta_{2}+\Delta_{3}=d\right)$,

$$
\int d^{d} x_{4} \frac{\gamma_{\mu} x_{41}^{\mu} \gamma_{\nu} x_{24}^{\nu}}{\left|x_{14}\right|^{2 \Delta_{1}+1}\left|x_{24}\right|^{2 \Delta_{2}+1}\left|x_{34}\right|^{2 \Delta_{3}}}=\frac{\pi^{\frac{d}{2}} A\left(\Delta_{3}\right) V\left(\Delta_{1}, \Delta_{2}\right) \gamma_{\mu} x_{31}^{\mu} \gamma_{\nu} x_{23}^{\nu}}{\left|x_{12}\right|^{d-2 \Delta_{3}}\left|x_{13}\right|^{d-2 \Delta_{2}+1}\left|x_{23}\right|^{d-2 \Delta_{1}+1}},
$$

or diagrammatically,

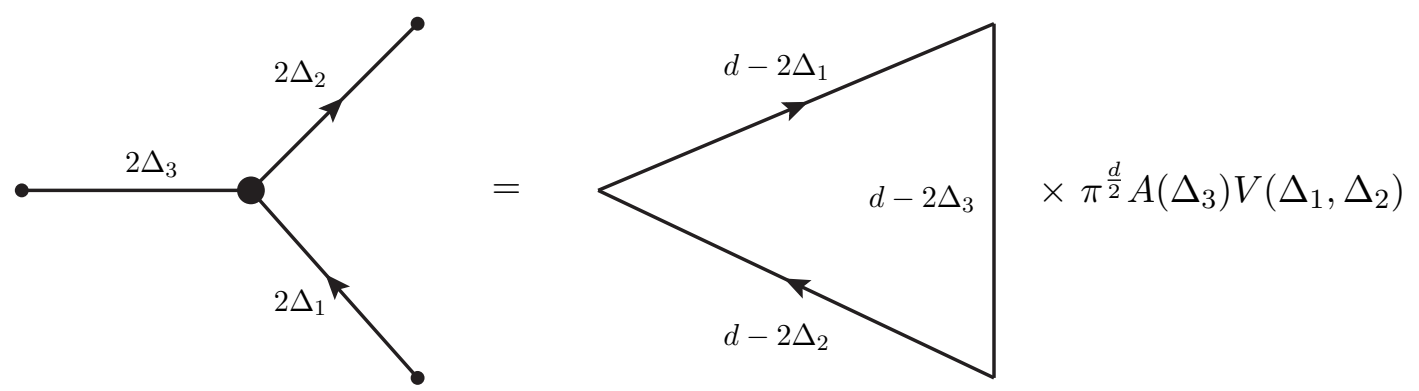

\subsection{Propagators at the next-to-leading order}

We now review the formalism to reproduce the leading order anomalous dimensions of the fields $s$ and $\psi$, as well as the $1 / N$ corrections to the amplitudes of their propagators [34, 42]. These results will be used extensively in the next section.

For the Dirac fermion the relevant conformal graph is,

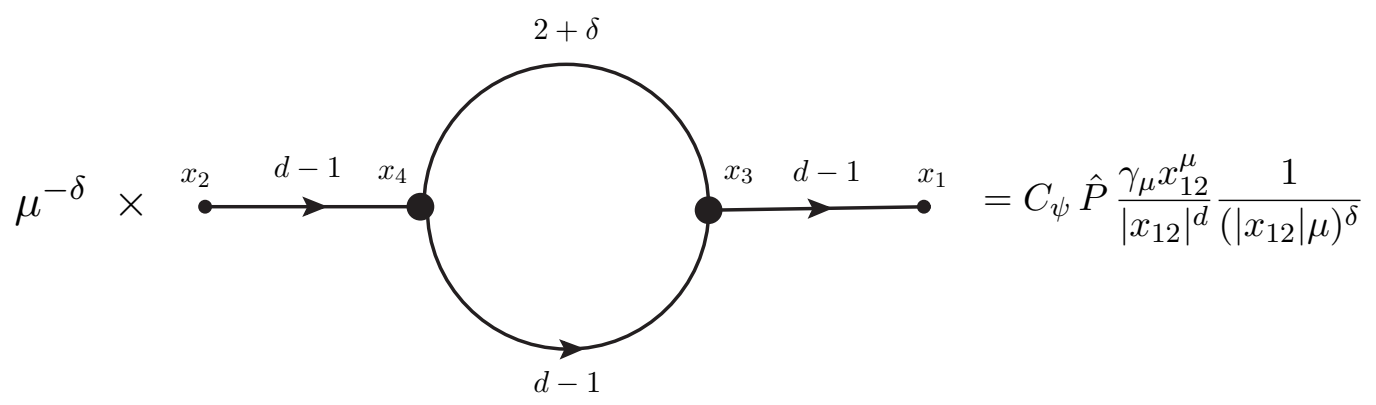

This diagram diverges; we regularized it by introducing a slight shift $\delta$ in the scaling dimension of $s$ and an arbitrary scale $\mu$, to maintain correct dimensionality [1, 45]. The 
integrals over internal vertices $x_{3,4}$ can be done by using the propagator merging relations reviewed above. Expanding around $\delta=0$ yields,

$$
\hat{P}=\frac{2 \gamma_{\psi}}{\delta}+A_{\psi},
$$

where $[34,42]$,

$$
\begin{aligned}
\gamma_{\psi} & =-\frac{1}{N} \frac{2^{d-1} \sin \left(\frac{\pi d}{2}\right) \Gamma\left(\frac{d-1}{2}\right)}{\pi^{3 / 2} d \Gamma\left(\frac{d}{2}-1\right)}, \\
A_{\psi} & =-\frac{2}{d} \gamma_{\psi} .
\end{aligned}
$$

Similarly, one can derive the regularized $s$ field propagator [42]

$$
\langle s(x) s(0)\rangle=C_{s} \frac{1}{|x|^{2}} \frac{1}{(|x| \mu)^{\delta}}\left(1+\frac{2 \gamma_{s}}{\delta}+A_{s}\right),
$$

with

$$
\begin{aligned}
\gamma_{s} & =\frac{1}{N} \frac{4 \sin \left(\frac{\pi d}{2}\right) \Gamma(d)}{\pi d \Gamma\left(\frac{d}{2}\right)^{2}}=-4 \frac{d-1}{d-2} \gamma_{\psi} \\
A_{s} & =-\left(H_{d-2}+\frac{2}{d}+\pi \cot \left(\frac{\pi d}{2}\right)\right) \gamma_{s},
\end{aligned}
$$

where $H_{n}$ is the $n$ 'th harmonic number.

To remove the $1 / \delta$ poles in the above propagators, one has to renormalize the fields,

$$
\psi \rightarrow \sqrt{1+\frac{2 \gamma_{\psi}}{\delta}} \psi, \quad s \rightarrow \sqrt{1+\frac{2 \gamma_{s}}{\delta}} s .
$$

As a result, the physical correlators take the form, ${ }^{12}$

$$
\begin{aligned}
\langle\psi(x) \bar{\psi}(0)\rangle & =C_{\psi}\left(1+A_{\psi}\right) \mu^{-2 \gamma_{\psi}} \frac{x^{\mu} \gamma_{\mu}}{|x|^{2\left(\Delta_{\psi}+\gamma_{\psi}\right)+1}}, \\
\langle s(x) s(0)\rangle & =C_{s}\left(1+A_{s}\right) \mu^{-2 \gamma_{s}} \frac{1}{|x|^{2\left(\Delta_{s}+\gamma_{s}\right)}} .
\end{aligned}
$$

Notice that the field strength renormalization (4.17) induces a counterterm, which at the next-to-leading order in $1 / N$ is given by,

$$
\frac{1}{\sqrt{N}} \bar{\psi} \psi s \rightarrow \frac{1}{\sqrt{N}} \bar{\psi} \psi s+\frac{2 \gamma_{\psi}+\gamma_{s}}{\delta} \frac{1}{\sqrt{N}} \bar{\psi} \psi s .
$$

Or, equivalently,

$$
S_{\text {int }}^{\text {c.t. }}=\frac{2 \gamma_{\psi}+\gamma_{s}}{\delta} \frac{1}{\sqrt{N}} \int d^{d} x \bar{\psi} \psi s .
$$

\footnotetext{
${ }^{12}$ In order to avoid cluttering the notation, we do not distinguish between physical and bare fields.
} 


\subsection{Gross-Neveu-Yukawa model}

Before closing this section, we briefly review the relation between the Gross-Neveu and Gross-Neveu-Yukawa (GNY) models. The results reviewed here are used to check our calculations in the next section.

The GNY model is defined by the action,

$$
S=\int d^{d} x\left(\bar{\psi} \gamma^{\mu} \partial_{\mu} \psi+g_{1} \bar{\psi} \psi s+\frac{1}{2}(\partial s)^{2}+\frac{g_{2}}{24} s^{4}\right)
$$

This model is manifestly renormalizable in $d \leq 4$, because the couplings $g_{1}$ and $g_{2}$ are relevant at the Gaussian fixed point in $d<4$ and marginally irrelevant in $d=4$. Below four dimensions they flow to an interacting fixed point in the IR limit. The critical values of the couplings can be calculated by using the standard $\epsilon$-expansion in $d=4-\epsilon$ dimensions [15],

$$
\begin{aligned}
& g_{1}^{\star}=4 \pi \sqrt{\frac{\epsilon}{N+6}}+\mathcal{O}(\epsilon)=4 \pi \sqrt{\frac{\epsilon}{N}}\left(1-\frac{3}{N}+\mathcal{O}\left(1 / N^{2}\right)\right)+\mathcal{O}(\epsilon), \\
& g_{2}^{\star}=\frac{384 \pi^{2} \epsilon N}{(N+6)\left(N-6+\sqrt{N^{2}+132 N+36}\right)} .
\end{aligned}
$$

Furthermore, the scaling dimensions of the fields at the fixed point were calculated in [15] (see [46] for a comprehensive review),

$$
\Delta_{\psi}^{(4-\epsilon)}=\frac{3}{2}-\frac{N+4}{2(N+6)} \epsilon+\mathcal{O}\left(\epsilon^{2}\right) \quad \Delta_{s}^{(4-\epsilon)}=1-\frac{3}{N+6} \epsilon+\mathcal{O}\left(\epsilon^{2}\right) .
$$

This formula shows that in the large $N$ limit $\Delta_{s}^{(4-\epsilon)}=1+\mathcal{O}(1 / N)$, up to linear order in $\epsilon$. In fact, a general argument based on the $1 / N$ expansion shows that this relation is true for any $2 \leq d \leq 4$, see, e.g., [46]. In particular, the operators $(\partial s)^{2}$ and $s^{4}$ are irrelevant in the large $N$ limit below four dimensions, because their scaling dimension approaches 4 in this limit. Therefore, the effective action of the GNY model in the deep IR matches that of the GN model in the deep UV, and the two models are described by the same CFT at their respective fixed points [15, 17, 47, 48].

As was pointed out in [15], the equivalence between the GN and the GNY models is analogous to the equivalence between the critical $\phi^{4}$ vector model and the non-linear sigma model. A similar equivalence, albeit one that is slightly less exhaustive, also holds between the critical $\phi^{4}$ vector model in $4<d \leq 6$ and the model of $N+1$ massless scalars with cubic interaction $[17,18,49] .^{13}$

As an illustration of the equivalence between the critical GN and GNY models, let us consider the 1PI vertices for the field $s$. To leading order in the $1 / N$ expansion, they are represented diagrammatically by a single fermionic loop with a given number of $s$ legs attached to it. Because of the equivalence, vertices with the same number and type of legs

\footnotetext{
${ }^{13}$ It was argued in these works that the equivalence between the models in $d=6-\epsilon$ dimensions holds up to $\mathcal{O}\left(\epsilon^{4}\right)$ order.
} 
in both models should match, ${ }^{14}$ i.e. for $n$ external fields $s$, we have

$$
\begin{aligned}
& \left(-\frac{1}{\sqrt{N}}\right)^{n} C_{s}^{\frac{n}{2}} C_{\psi}^{n} \int \prod_{i=1}^{n} d^{d} x_{i} s_{i} \mathcal{I}_{n}\left(x_{1}, \ldots, x_{n}\right) \\
& =\left(-g_{1}^{\star}\right)^{n} \tilde{C}_{s}^{\frac{n}{2}} C_{\psi}^{n} \int \prod_{i=1}^{n} d^{d} x_{i} s_{i} \mathcal{I}_{n}\left(x_{1}, \ldots, x_{n}\right),
\end{aligned}
$$

where the two sides of this equation represent the same vertex in the GN and GNY models respectively, $\mathcal{I}_{n}\left(x_{1}, \ldots, x_{n}\right)$ corresponds to the internal $\psi$-loop, and the external fields $s$ are normalized such that the amplitude of their propagators equals unity. In particular, $\tilde{C}_{s}=$ $\Gamma\left(\frac{d}{2}-1\right) /\left(4 \pi^{\frac{d}{2}}\right)$, because the field $s$ in the GNY action (4.22) is canonically normalized. Using (4.7) and (4.23), one can verify that the above identity indeed holds to leading order in the $\epsilon$ and $1 / N$ expansion.

Moreover, the symmetry pattern of the models match. The actions (4.2) and (4.22) are invariant under the discrete symmetry $[14,15,46]$

$$
\begin{aligned}
\left(x^{1}, \ldots, x^{\mu-1}, x^{\mu}, x^{\mu+1}, \ldots, x^{d}\right) & \rightarrow\left(x^{1}, \ldots, x^{\mu-1},-x^{\mu}, x^{\mu+1}, \ldots, x^{d}\right), \\
s \rightarrow-s, \quad \psi & \rightarrow \gamma_{\mu} \psi, \quad \bar{\psi} \rightarrow-\bar{\psi} \gamma_{\mu} .
\end{aligned}
$$

Notice that such a transformation leaves the fermionic kinetic term invariant while it transforms $\bar{\psi} \psi \rightarrow-\bar{\psi} \psi$, in analogy with the chiral transformation in even dimensions and parity in odd dimensions. Thus, for instance, any correlator with an odd number of fields $s$ must vanish. The three point function $\langle s s s\rangle$ is the simplest instance where one can directly see that the correlator vanishes. To leading order in the large- $N$ expansion, it is given by,

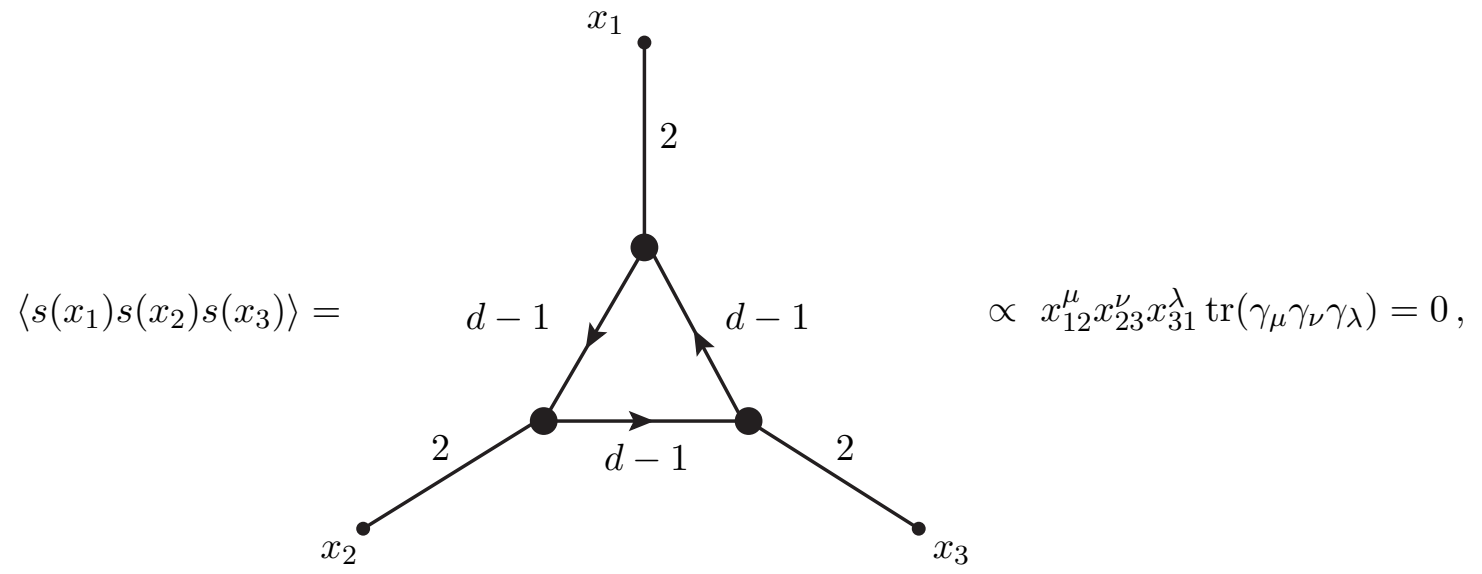

where the last equality is true in any integer dimension $d=2,3,4$, whereas in general $2<d<4$ it holds by analytic continuation [41]. ${ }^{15}$

In contrast, the three-point function $\langle\bar{\psi} \psi s\rangle$ is nontrivial. To leading order in $\epsilon$ it can be derived using the tree level cubic interaction and the star-triangle relation (4.10),

$$
\left\langle\bar{\psi}\left(x_{1}\right) \psi\left(x_{2}\right) s\left(x_{3}\right)\right\rangle=\tilde{C}_{\bar{\psi} \psi s} \frac{\gamma_{\mu} x_{13}^{\mu} \gamma_{\nu} x_{32}^{\nu}}{\left|x_{12}\right|^{d-2 \Delta_{s}}\left|x_{13}\right|^{d-2 \Delta_{\psi}+1}\left|x_{23}\right|^{d^{-2 \Delta_{\psi}+1}}},
$$

\footnotetext{
${ }^{14} \mathrm{~A}$ similar correspondence holds between the critical $\phi^{4}$ vector model and the model of $N+1$ massless scalars with cubic interaction [50].

${ }^{15}$ The trace of the gamma matrices vanishes identically in even dimensions. In $d=3, \operatorname{tr}\left(\gamma_{\mu} \gamma_{\nu} \gamma_{\lambda}\right) \propto \epsilon^{\mu \nu \lambda}$.
} 
where the OPE coefficient is given by

$$
\tilde{C}_{\bar{\psi} \psi s}=-g_{1}^{\star} C_{\psi} \tilde{C}_{s}^{\frac{1}{2}} \pi^{\frac{d}{2}} A(1) V\left(\frac{d-1}{2}, \frac{d-1}{2}\right)=-\sqrt{\frac{\epsilon}{N}}\left(1-\frac{3}{N}+\mathcal{O}\left(1 / N^{2}\right)\right) .
$$

Note that, by definition of the OPE coefficient, all the fields are normalized so that their two-point functions have unit amplitude.

\section{CFT data for the critical Gross-Neveu model}

In this section we use the background field method [29] to derive some CFT data for the critical Gross-Neveu model. Specifically, we calculate the three-point function $\left\langle\bar{\psi}\left(x_{1}\right) \psi\left(x_{2}\right) s\left(x_{3}\right)\right\rangle$ and the associated conformal effective vertex, up to next-to-leading order in the $1 / N$ expansion. In addition, we calculate the leading order OPE coefficient determined by the correlation function $\left\langle\bar{\psi}\left(x_{1}\right) \psi\left(x_{2}\right) s^{2}\left(x_{3}\right)\right\rangle$. Our calculations hold in general $2 \leq d \leq 4$, and the final results agree with their counterparts obtained through the $\epsilon$-expansion of the critical Gross-Neveu-Yukawa model in the vicinity of $d=4$. The latter serves as a check of our calculations, since the two critical models are equivalent [15].

Evaluation of $\left\langle\bar{\psi}\left(x_{1}\right) \psi\left(x_{2}\right) s\left(x_{3}\right)\right\rangle$. In what follows, the amplitudes of the two-point functions are normalized to unity, i.e. we rescale the fields,

$$
\psi \rightarrow \sqrt{C_{\psi}\left(1+A_{\psi}\right)} \psi, \quad s \rightarrow \sqrt{C_{s}\left(1+A_{s}\right)} s .
$$

The leading order three-point function for the normalized fields, which can be derived by using the tree level cubic vertex and the star-triangle relation, reviewed in section 4.1, is,

$$
\left\langle\bar{\psi}\left(x_{1}\right) \psi\left(x_{2}\right) s\left(x_{3}\right)\right\rangle=C_{\bar{\psi} \psi s} \frac{\gamma_{\mu} x_{13}^{\mu} \gamma_{\nu} x_{32}^{\nu}}{\left|x_{12}\right|^{d-2 \Delta_{s}}\left|x_{13}\right|^{d-2 \Delta_{\psi}+1}\left|x_{23}\right|^{d-2 \Delta_{\psi}+1}},
$$

where the leading order OPE coefficient is given by,

$$
C_{\bar{\psi} \psi s}=-\frac{1}{\sqrt{N}} C_{\psi} C_{s}^{\frac{1}{2}} \pi^{\frac{d}{2}} A(1) V\left(\frac{d-1}{2}, \frac{d-1}{2}\right)=\frac{-2^{\frac{d}{2}}}{\sqrt{N}(d-2) \pi^{\frac{3}{4}}} \sqrt{-\frac{\sin \left(\frac{\pi d}{2}\right) \Gamma\left(\frac{d-1}{2}\right)}{\Gamma\left(\frac{d}{2}-1\right)}} .
$$

Loops are associated with higher order terms in $1 / N$, which modify (5.2). However, conformal invariance together with the discrete symmetry (4.27) fixes the structure of the full three-point function. In our case, it takes the form, ${ }^{16}$

$$
\left\langle\bar{\psi}\left(x_{1}\right) \psi\left(x_{2}\right) s\left(x_{3}\right)\right\rangle=C_{\bar{\psi} \psi s}\left(1+W_{\bar{\psi} \psi s}\right) \frac{\mu^{-2 \gamma_{\psi}-\gamma_{s}} \gamma_{\mu} x_{13}^{\mu} \gamma_{\nu} x_{32}^{\nu}}{\left|x_{13}\right|^{\Delta_{s}+\gamma_{s}+1}\left|x_{32}\right|^{\Delta_{s}+\gamma_{s}+1}\left|x_{12}\right|^{2 \Delta_{\psi}-\Delta_{s}+2 \gamma_{\psi}-\gamma_{s}}},
$$

\footnotetext{
${ }^{16}$ Conformal symmetry uniquely determines the three point function of two fermion and one scalar field in terms of two possible structures [51, 52]. However, in our case the discrete symmetry (4.27) eliminates one of them, and the end result is (5.4). More generally, $\left\langle s^{2 k+1} \psi \bar{\psi}\right\rangle$ and $\left\langle s^{2(k+1)} \psi \bar{\psi}\right\rangle$ for $k=0,1,2, \ldots$ have the same form as (4.27) and (5.24) respectively, see also [53] where $\left\langle s^{2} \psi \bar{\psi}\right\rangle$ was calculated at the next-to-leading order in $1 / N$.
} 
where the anomalous dimensions and $W_{\bar{\psi} \psi s}$ encompass loop corrections to the three-point function (5.2).

The anomalous dimensions $\gamma_{s}$ and $\gamma_{\psi}$ were evaluated in the previous section; in this section our goal is to determine $W_{\bar{\psi} \psi s}$. To this end, we need to dress the tree level diagram with $1 / N$ corrections. As we will see below, $W_{\bar{\psi} \psi s}$ receives contributions from the $1 / N$ corrections to the amplitudes of external propagators, as well as from the $1 / N$ corrections to the cubic vertex itself. The former corrections were evaluated in the previous section $\left(A_{s}\right.$ and $\left.A_{\psi}\right)$, so we only need to evaluate the latter terms.

The full cubic vertex of the effective action is non-local. By conformal invariance its form is fixed up to an overall constant prefactor, and can be represented diagrammatically as a conformal triangle [54],

$$
-\frac{\hat{Z} \mu^{2 \gamma_{\psi}+\gamma_{s}}}{\sqrt{N}} \times \underbrace{2 \alpha}_{2 \beta}=-\frac{\hat{Z}}{\sqrt{N}} \iiint_{\psi\left(x_{2}\right)}^{s\left(x_{3}\right)} \frac{\mu^{2 \gamma_{\psi}+\gamma_{s}} x_{13}^{\mu} \gamma_{\mu} x_{32}^{\nu} \gamma_{\nu}}{\left|x_{13}\right|^{2 \alpha+1}\left|x_{32}\right|^{2 \alpha+1}\left|x_{12}\right|^{2 \beta}} \bar{\psi}\left(x_{1}\right) \psi\left(x_{2}\right) s\left(x_{3}\right),
$$

where the unknown constant $\hat{Z}$ is closely related to $W_{\bar{\psi} \psi s}$, while $\alpha$ and $\beta$ are determined by noticing that each vertex, denoted by a solid dot in the above graph, should be conformal after attaching to it an appropriate full propagator (4.18) or (4.19). Hence,

$$
\alpha=\Delta_{\psi}-\frac{\gamma_{s}}{2}, \quad \beta=\Delta_{s}-\gamma_{\psi}+\frac{\gamma_{s}}{2} .
$$

We now focus on calculating the only remaining unknown, $\hat{Z}$. By definition, it is determined by the following diagrammatic equation, which represents the perturbative expansion of the three-point function $\left\langle\bar{\psi}\left(x_{1}\right) \psi\left(x_{2}\right) s\left(x_{3}\right)\right\rangle$,
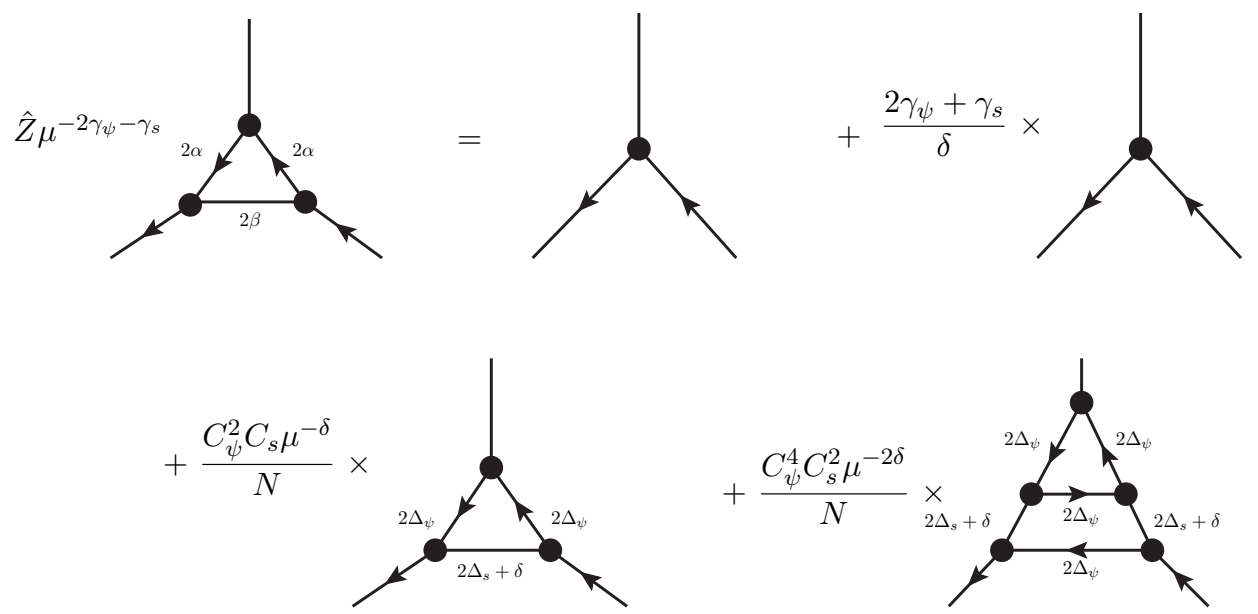

where the external legs are not amputated; they correspond to the full propagators (4.18) and (4.19).

The first graph on the right-hand side represents the cubic interaction of (4.2). The rest of the terms reveal the $1 / N$ terms. Specifically, the two diagrams on the second line 
of (5.7) account for the loop corrections to the leading order cubic vertex. In fact, the last diagram is trivial, because it contains a vanishing $\langle s s s\rangle$ sub-graph, discussed in section 4.3. On the other hand, the first diagram on the second line of (5.7) results in a non-trivial next-to-leading order contribution to the cubic vertex. ${ }^{17}$ This diagram diverges and can be regularized by a small shift $\delta$ in the scaling dimension of $s$. This is the same regulator $\delta$ used in section 4.2 to calculate the $1 / N$ corrections to the propagators of the fields $\psi$ and $s$.

While the couplings are not renormalized at the fixed point, there is still renormalization of the fields. In particular, the second diagram on the right-hand side of (5.7) is associated with the counterterm (4.21) induced by wave function renormalization. In the limit $\delta \rightarrow 0$, this counterterm cancels the divergences of the loop diagrams.

The diagrammatic equation (5.7) simplifies if the external propagator of the auxiliary field $s$ is replaced with a constant background $\bar{s}$. At the level of the path integral, this is tantamount to decomposing the Hubbard-Stratonovich field into a fluctuating component $s$ and a constant background $\bar{s}$, i.e. $s \rightarrow \bar{s}+s$. The resulting diagrammatic equation represents a linear (in $\bar{s}$ ) correction to the two-point function $\left\langle\bar{\psi}\left(x_{1}\right) \psi\left(x_{2}\right)\right\rangle_{\bar{s}}$ in the presence of a constant background,

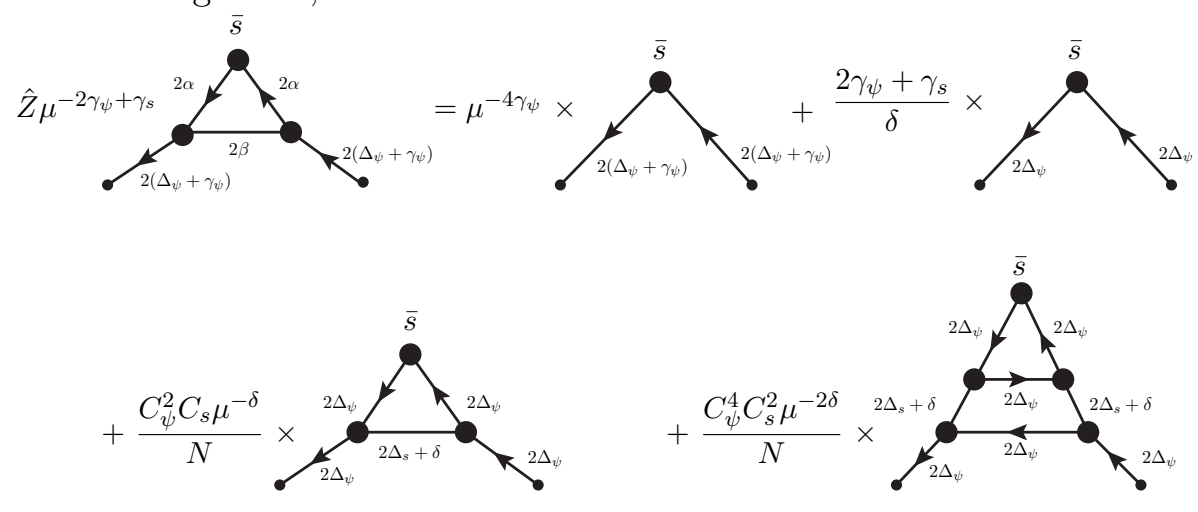

Note that the amplitudes of the external fermionic propagators (4.18) cancel on both sides of this equation, and we ignore them in what follows.

Each diagram in (5.8) can be calculated by repeatedly applying the fermion-fermion and fermion-scalar propagator merging relations reviewed in section 4.1. The calculation of each diagram starts by integrating over the insertion point of the vertex with a constant background $\bar{s}$. In particular, the left-hand side of (5.8) takes the form,

$$
\begin{aligned}
\text { l.h.s. of }(5.8)= & \pi^{\frac{3 d}{2}} \hat{Z} A\left(1+\gamma_{s}\right) V\left(\frac{d-1-\gamma_{s}}{2}, \frac{d-1-\gamma_{s}}{2}\right) \\
& \times A\left(\frac{d-\gamma_{s}}{2}-\gamma_{\psi}\right) V\left(\frac{d-1}{2}+\gamma_{\psi}, \frac{1+\gamma_{s}}{2}\right) \\
& \times A\left(1-\gamma_{\psi}+\frac{\gamma_{s}}{2}\right) V\left(\frac{d-1-\gamma_{s}}{2}, \frac{d-1}{2}+\gamma_{\psi}\right) \frac{\mu^{-2 \gamma_{\psi}+\gamma_{s}} \bar{s}}{\left|x_{12}\right|^{d-2+2 \gamma_{\psi}-\gamma_{s}}} .
\end{aligned}
$$

\footnotetext{
${ }^{17}$ The two last graphs of (5.7) are analogous to the corresponding vertex diagrams in the $O(N)$ vector model. However, unlike the case studied here, in the critical $O(N)$ vector model both diagrams give rise to a non-trivial contribution, see, e.g., [50] for a recent discussion. In appendix A we recover $\hat{Z}$ in the vector model using the background field method.
} 
Substituting

$$
\hat{Z}=Z_{0}(1+\delta Z),
$$

where $\delta Z \sim \mathcal{O}(1 / N)$, and expanding in the small parameters $\gamma_{s}, \gamma_{\psi} \sim \mathcal{O}(1 / N)$, yields

$$
\begin{aligned}
\text { l.h.s. of }(5.8)= & \frac{8 \pi^{\frac{3 d}{2}} Z_{0}}{(d-2)^{2} \Gamma\left(\frac{d}{2}\right)^{3}\left(\gamma_{s}+2 \gamma_{\psi}\right)} \frac{\bar{s}}{\left|x_{12}\right|^{d-2}} \\
& \times\left(1+\delta Z+\frac{3 \gamma_{s}-2 \gamma_{\psi}}{d-2}-\left(2 \gamma_{\psi}-\gamma_{s}\right) \log \left(\left|x_{12}\right| \mu\right)\right) .
\end{aligned}
$$

On the other hand, this expression is equal to the sum of the three diagrams on the right-hand side of (5.8), which we denote by, ${ }^{18}$

$$
\text { r.h.s. of }(5.8)=v_{\text {tree }}+v_{\text {c.t. }}+v_{\text {loop }}(\delta) \text {. }
$$

The tree level term is given by,

$$
v_{\text {tree }}=-\pi^{\frac{d}{2}} A\left(1-2 \gamma_{\psi}\right) V\left(\frac{d-1}{2}+\gamma_{\psi}, \frac{d-1}{2}+\gamma_{\psi}\right) \frac{\mu^{-4 \gamma_{\psi}} \bar{s}}{\left|x_{12}\right|^{d-2+4 \gamma_{\psi}}} .
$$

It contributes to the leading and subleading orders in $1 / N$,

$$
\begin{aligned}
v_{\text {tree }}= & -\frac{2 \pi^{d / 2}}{(d-2) \Gamma\left(\frac{d}{2}\right)}\left(1+\frac{1}{N} \frac{4 \sin \left(\frac{\pi d}{2}\right) \Gamma(d-1)}{\pi d \Gamma\left(\frac{d}{2}\right)^{2}}-4 \gamma_{\psi} \log \left(\left|x_{12}\right| \mu\right)\right) \\
& \times \frac{\bar{s}}{\left|x_{12}\right|^{d-2}}+\mathcal{O}\left(1 / N^{2}\right) .
\end{aligned}
$$

The counterterm satisfies

$$
v_{\text {c.t. }}=-\frac{2 \pi^{d / 2}}{(d-2) \Gamma\left(\frac{d}{2}\right)} \frac{2 \gamma_{\psi}+\gamma_{s}}{\delta} \frac{\bar{s}}{\left|x_{12}\right|^{d-2}} .
$$

Finally, the loop diagram takes the form

$$
\begin{aligned}
v_{\text {loop }}(\delta)= & \frac{C_{\psi}^{2} C_{s}}{N} \frac{\bar{s}}{\left(\mu\left|x_{12}\right|\right)^{d-2+\delta}} \pi^{\frac{3 d}{2}} A(1) V\left(\frac{d-1}{2}, \frac{d-1}{2}\right) A\left(\frac{d+\delta}{2}\right) \\
& \times V\left(\frac{d-1}{2}, \frac{1-\delta}{2}\right) A\left(1-\frac{\delta}{2}\right) V\left(\frac{d-1+\delta}{2}, \frac{d-1}{2}\right) .
\end{aligned}
$$

Expanding around $\delta=0$ yields,

$$
\begin{aligned}
v_{\text {loop }}(\delta) & =-\frac{2 \pi^{d / 2}}{(d-2) \Gamma\left(\frac{d}{2}\right)} \frac{\bar{s}}{\left|x_{12}\right|^{d-2}} \\
& \times\left(-\frac{2 \gamma_{\psi}+\gamma_{s}}{\delta}+\left(2 \gamma_{\psi}+\gamma_{s}\right) \log \left(\left|x_{12}\right| \mu\right)+\frac{1}{N} \frac{2^{d-1} \sin \left(\frac{\pi d}{2}\right) \Gamma\left(\frac{d-1}{2}\right)}{\pi^{3 / 2}(d-2) \Gamma\left(\frac{d}{2}\right)}\right) .
\end{aligned}
$$

\footnotetext{
${ }^{18}$ Recall that the last diagram in (5.8) vanishes, because it has a subdiagram $\langle s s s\rangle$ which does not respect $s \rightarrow-s$ symmetry.
} 
As expected, the sum of the three terms (5.14), (5.15) and (5.17) is finite in the limit $\delta \rightarrow 0$

$$
\begin{aligned}
\text { r.h.s. of }(5.8)= & v_{\text {tree }}+v_{\text {c.t. }}+v_{\text {loop }}(\delta) \underset{\delta \rightarrow 0}{\rightarrow}-\frac{2 \pi^{d / 2}}{(d-2) \Gamma\left(\frac{d}{2}\right)} \frac{\bar{s}}{\left|x_{12}\right|^{d-2}} \\
& \times\left(1+\frac{1}{N} \frac{2(3 d-4) \sin \left(\frac{\pi d}{2}\right) \Gamma(d-1)}{\pi(d-2) d \Gamma\left(\frac{d}{2}\right)^{2}}-\left(2 \gamma_{\psi}-\gamma_{s}\right) \log \left(\left|x_{12}\right| \mu\right)\right) .
\end{aligned}
$$

Comparing (5.11) to (5.18), we get $\hat{Z}(5.10)$,

$$
\begin{aligned}
Z_{0} & =-\frac{(d-2) \Gamma\left(\frac{d}{2}\right)^{2}}{4 \pi^{d}}\left(2 \gamma_{\psi}+\gamma_{s}\right) \\
\delta Z & =-\frac{8 \Gamma(d) \sin \left(\frac{\pi d}{2}\right)}{N d(d-2) \pi \Gamma\left(\frac{d}{2}\right)^{2}}=-\frac{2}{d-2} \gamma_{s} .
\end{aligned}
$$

The same result was recently obtained in [42], using a different method.

Finally, calculating the three-point function $\left\langle\bar{\psi}\left(x_{1}\right) \psi\left(x_{2}\right) s\left(x_{3}\right)\right\rangle$ simply involves attaching the full propagators (4.18), (4.19) to the effective cubic vertex (5.5), and integrating over the internal vertices through the use of the star-triangle relation (4.10). After normalizing the external fields as in (5.1), the final result reads,

$$
\begin{aligned}
\left\langle\bar{\psi}\left(x_{1}\right) \psi\left(x_{2}\right) s\left(x_{3}\right)\right\rangle= & -\frac{C_{\psi}\left(1+A_{\psi}\right) \sqrt{C_{s}\left(1+A_{s}\right)}}{\sqrt{N}} \\
& \times \frac{\hat{Z} U \mu^{-2 \gamma_{\psi}-\gamma_{s}} \gamma_{\mu} x_{13}^{\mu} \gamma_{\nu} x_{32}^{\nu}}{\left|x_{13}\right|^{\Delta_{s}+\gamma_{s}+1}\left|x_{32}\right|^{\Delta_{s}+\gamma_{s}+1}\left|x_{12}\right|^{2 \Delta_{\psi}-\Delta_{s}+2 \gamma_{\psi}-\gamma_{s}}},
\end{aligned}
$$

where

$$
\begin{aligned}
U= & -\pi^{\frac{3 d}{2}} A\left(1+\gamma_{s}\right) V\left(\frac{d-1-\gamma_{s}}{2}, \frac{d-1-\gamma_{s}}{2}\right) A\left(\frac{d-\gamma_{s}}{2}-\gamma_{\psi}\right) \\
& \times V\left(\frac{1+\gamma_{s}}{2}, \frac{d-1}{2}+\gamma_{\psi}\right) A\left(1-\gamma_{\psi}+\frac{\gamma_{s}}{2}\right) V\left(\frac{d-1-\gamma_{s}}{2}, \frac{d-1}{2}+\gamma_{\psi}\right) \\
= & -\frac{8 \pi^{\frac{3 d}{2}}}{(d-2)^{2} \Gamma\left(\frac{d}{2}\right)^{3}\left(\gamma_{s}+2 \gamma_{\psi}\right)}\left(1+\delta U+\mathcal{O}\left(1 / N^{2}\right)\right), \quad \delta U=\frac{3 \gamma_{s}-2 \gamma_{\psi}}{d-2} .
\end{aligned}
$$

In the last line we expanded in the small anomalous dimensions. Expanding the right-hand side of (5.21), up to next-to-leading order in $1 / N$, and comparing to (5.4), we deduce that the leading term reproduces (5.3), while the next-to-leading order correction is given by, ${ }^{19}$

$$
W_{\bar{\psi} \psi s}=A_{\psi}+\frac{A_{s}}{2}+\delta Z+\delta U=\frac{2(d-1)\left((d-2) H_{d-2}+\pi(d-2) \cot \left(\frac{\pi d}{2}\right)-2\right)}{(d-2)^{2}} \gamma_{\psi} .
$$

It is instructive to check our result for $W_{\bar{\psi} \psi_{s}}$. To this end, we substitute (5.3) and (5.23) into (5.4), and expand the three-point function $\langle\bar{\psi} \psi s\rangle$ in $\epsilon=4-d$. The leading order term in $\epsilon$ matches (4.29), in accord with the equivalence between the critical Gross-Neveu

\footnotetext{
${ }^{19}$ The analogous OPE coefficient in the $O(N)$ vector model was recently derived in [50].
} 


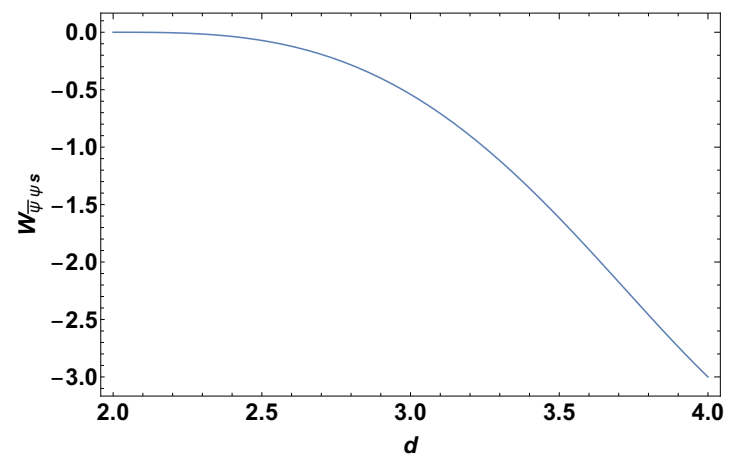

Figure 11. The $1 / N$ correction to the OPE coefficient, $W_{\bar{\psi} \psi_{s}}$ (defined in eq. 5.4), as a function of space-time dimension $2 \leq d \leq 4$. The end points of the plot satisfy $W_{\bar{\psi} \psi_{s}}(d=2)=0$ and $W_{\bar{\psi} \psi s}(d=4)=-3$.

and Gross-Neveu-Yukawa models. Moreover, $W_{\bar{\psi} \psi s}$ vanishes in $d=2$, and therefore the OPE coefficient equals -1 , up to order $1 / N$. This result is expected, because the critical GN model is free in two dimensions and $\langle\bar{\psi} \psi s\rangle \sim\langle\bar{\psi} \psi(\bar{\psi} \psi)\rangle$, since $s \sim \bar{\psi} \psi / \sqrt{N}$, by the equations of motion. In particular, it follows from the standard Wick contraction applied to a free field theory (with properly normalized fields) that the OPE coefficient is indeed -1 , to all orders in $1 / N$. Figure 11 displays $W_{\bar{\psi} \psi_{s}}$ for dimensions $2 \leq d \leq 4$.

Evaluation of $\left\langle\bar{\psi}\left(x_{1}\right) \psi\left(x_{2}\right) s^{2}\left(x_{3}\right)\right\rangle$. Let us close this section by deriving the threepoint function $\left\langle\bar{\psi} \psi s^{2}\right\rangle$ between the Dirac fields and the composite operator $s^{2}$. To leading order in the $1 / N$ expansion, this correlator is not affected by the anomalous dimensions of $\psi$ and $s^{2}$

$$
\left\langle s^{2}\left(x_{1}\right) \psi\left(x_{2}\right) \bar{\psi}\left(x_{3}\right)\right\rangle=C_{s^{2} \bar{\psi} \psi} \frac{x_{23}^{\mu} \gamma_{\mu}}{\left|x_{12}\right|^{2}\left|x_{13}\right|^{2}\left|x_{23}\right|^{d-2}}(1+\mathcal{O}(1 / N))
$$

On the other hand, it is determined by the diagram

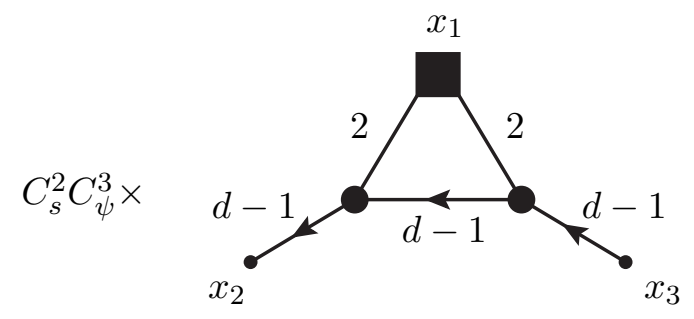

where insertion of $s^{2}$ is denoted by a black square.

Using the star-triangle relation (4.10) to integrate over the internal vertices, and normalizing $s^{2}$ and the Dirac fields according to $s^{2} \rightarrow \sqrt{2} C_{s} s^{2}$ and (5.1), respectively, yields

$$
C_{s^{2} \bar{\psi} \psi}=\frac{1}{N} \frac{2^{d-\frac{3}{2}} \sin \left(\frac{\pi d}{2}\right) \Gamma\left(\frac{d-1}{2}\right)}{\pi^{3 / 2} \Gamma\left(\frac{d}{2}\right)}
$$


This result is in full agreement with the GNY model. ${ }^{20}$ Moreover, the three point function (5.24) respects discrete symmetry (4.27). Indeed,

$$
\left\langle s\left(x_{1}\right)^{2} \psi\left(x_{2}\right) \bar{\psi}\left(x_{3}\right)\right\rangle \rightarrow-\gamma^{\mu}\left\langle s\left(x_{1}\right)^{2} \psi\left(x_{2}\right) \bar{\psi}\left(x_{3}\right)\right\rangle \gamma^{\mu} \quad \Rightarrow \quad C_{s^{2} \bar{\psi} \psi} \rightarrow C_{s^{2} \bar{\psi} \psi}
$$

\section{Discussion}

In this paper we used the background field method [29] as a simple way to calculate conformal cubic vertices in critical $\mathcal{O}(N)$ vector models, up to next-to-leading order in $1 / N$. To the best of our knowledge, this method of calculation has not been previously employed in large- $N$ conformal perturbation theory.

We explicitly studied two critical vector models: the $O(N)$ sextic $\phi^{6}$ model in dimension $d=3$, and the $U(n)$ Gross-Neveu model in dimensions $2 \leq d \leq 4$. For the $\phi^{6}$ model, we derived the leading order anomalous dimensions, (2.31) and (3.10), of the composite operators $\rho^{n} \sim\left(\phi^{2}\right)^{n}$, as well as the OPE coefficients associated with the three-point functions $\left\langle\phi^{2} \phi^{2} \phi^{2}\right\rangle$ and $\left\langle\phi \phi \phi^{2}\right\rangle$, up to the next-to-leading order in $1 / N$, see (3.17) and (3.19). For the critical Gross-Neveu model, we derived the leading order and next-to-leading order OPE coefficient of the three-point functions $\langle\bar{\psi} \psi s\rangle$ and $\left\langle\bar{\psi} \psi s^{2}\right\rangle$, see (5.3), (5.23) and (5.25). Our results agree with the literature $[26,42]$ and extend it in several ways.

There are a number of motivations for studying critical vector models. The GrossNeveu model, specifically, is asymptotically safe despite being non-renormalizable in the vicinity of the Gaussian fixed point above two dimensions. Its RG flow is therefore completely determined by a finite number of parameters, and its UV fixed point has been extensively studied in the large- $N$ limit $[15,34-42,47]$. In particular, it was shown that the UV CFT of the model is equivalent to an IR CFT of the renormalizable Gross-NeveuYukawa model [15]. From this perspective, the Gross-Neveu model provides a laboratory where theoretical questions about the structure of RG flows and mechanisms of UV completion can be explicitly addressed. In two dimensions it presents a simple example of an asymptotically free field theory [14].

In contrast, the $\phi^{6}$ vector model is manifestly renormalizable in three dimensions, yet it also possesses a UV fixed point which is interesting in its own way. It turns out that the UV physics of the model is closely related to the study of big crunch singularities in asymptotically $A d S_{4}$ spacetimes [56, 57], see also [58]. In the latter context, one studies a marginal triple trace deformation of ABJM theory [59], where the deformation corresponds to adding a potential which is unbounded from below. The UV fixed point of the deformed ABJM theory in the limit of zero 't Hooft coupling is described by the critical $\phi^{6}$ model studied in this work. It would be interesting to systematically explore the effects of the non-perturbative instability in $1 / N$ [30] on the CFT data of the critical $\phi^{6}$ model and understand the cosmological implications of our findings in the holographic context studied in $[56,57]$.

\footnotetext{
${ }^{20}$ In the GNY model $\left\langle\bar{\psi} \psi s^{2}\right\rangle$ is determined by a similar diagram. To explicitly verify that it matches the GN counterpart, it is sufficient to use the leading order relation $\left(g_{1}^{\star}\right)^{2} \tilde{C}_{s} / C_{s}=1+\mathcal{O}(\epsilon, 1 / N)$, see section 4.3.
} 
It is tempting to study the critical $\phi^{6}$ vector model away from $d=3$. By continuity, the UV fixed point does not disappear as we move towards other dimensions, provided that $N$ is sufficiently large. Thus, we expect to generate a family of CFTs parameterized by $d$, and a natural question is whether there is a range for $d$ such that the corresponding CFTs are non-perturbatively stable.

Finally, it turns out that critical vector models exhibit peculiar behavior when coupled to a thermal bath. For instance, it was shown recently that there are $O(N)$ theories which have some of their internal symmetries broken at arbitrary finite temperature [60]. The results of this paper might be of help in understanding this behavior in three dimensions.

\section{Acknowledgments}

We thank Noam Chai, Soumyadeep Chaudhuri, Eliezer Rabinovici and Ritam Sinha for helpful discussions. The work of MG and MS is partially supported by the Binational Science Foundation (grant No. 2016186), the Israeli Science Foundation Center of Excellence (grant No. 2289/18) and by the Quantum Universe I-CORE program of the Israel Planning and Budgeting Committee (grant No. 1937/12). The work of VR is supported by NSF grant PHY-1911298, as well as the Sivian Fund of the IAS.

\section{A Interaction vertex in the critical $\phi^{4}$ model}

In this appendix we derive the effective interaction vertex in the critical $\phi^{4}$ vector model in $2 \leq d \leq 6$ dimensions. The $\phi^{4}$ vector model has an IR fixed point in dimensions $2 \leq d \leq 4$, and a UV fixed point in dimensions $4<d \leq 6$. For a recent discussion of this model see [50] and references therein, including [6, 7, 55, 61-67]. Our goal is to demonstrate that the background field method, used in section 5 to calculate the effective $\bar{\psi} \psi s$ vertex in the Gross-Neveu model, is also applicable to the $O(N)$ vector model. Our result for the cubic vertex in the $\phi^{4}$ model fully agrees with [55], where it was evaluated using a different method. Since, to the best of our knowledge, the background field method has not been used before in the context of large- $N$ conformal perturbation theory, we felt some researchers may find the current discussion useful. The discussion in this appendix parallels the discussion in section 5 .

The critical $\phi^{4}$ vector model is described by the action,

$$
S=\int d^{d} x\left(\frac{1}{2}(\partial \phi)^{2}+\frac{1}{\sqrt{N}} s \phi^{2}+\frac{2 \gamma_{\phi}+\gamma_{s}}{\sqrt{N} \delta} s \phi^{2}\right)
$$

where $\phi$ is a real $N$-component multiplet of scalar fields in the fundamental representation of $O(N), s$ is the auxiliary Hubbard-Stratonovich field, $\delta$ is a UV regulator, and $\gamma_{\phi, s}$ are the anomalous dimensions associated with the loop corrections to the large- $N$ scaling dimensions $\Delta_{\phi}=d / 2-1$ and $\Delta_{s}=2$ of $\phi$ and $s$, respectively. Note that the HubbardStratonovich field is dynamical; its propagator is built from an infinite series of $\phi$ bubbles. $^{21}$

\footnotetext{
${ }^{21}$ The effective action for $s$ includes an infinite tower of non-local $n$-vertices suppressed by $1 / N^{\frac{n}{2}-1}$.
} 
The UV divergences of the theory are regulated by adding a small shift to the scaling dimension of the internal $s$-lines in the Feynman graphs, i.e. $2 \Delta_{s} \rightarrow 2 \Delta_{s}+\delta$. In particular, the last term in (A.1) is the counterterm generated by wave function renormalization of the fields $\phi$ and $s$. Other possible counterterms vanish at the conformal fixed point.

The cubic term in the effective action will be non-local. By conformal invariance, its form is fixed up to an overall constant $\hat{Z}$, and can be represented diagrammatically as a conformal triangle [54],

$$
-\frac{2 \hat{Z} \mu^{2 \gamma_{\phi}+\gamma_{s}}}{\sqrt{N}} \times \underbrace{s\left(x_{3}\right)}_{2 \alpha}=-\frac{2 \hat{Z}}{\sqrt{N}} \iiint \frac{\mu^{2 \gamma_{\phi}+\gamma_{s}}}{\left|x_{13}\right|^{2 \alpha}\left|x_{32}\right|^{2 \alpha}\left|x_{12}\right|^{2 \beta}} \phi\left(x_{1}\right) \phi\left(x_{2}\right) s\left(x_{3}\right),
$$

where

$$
\alpha=\Delta_{\phi}-\frac{\gamma_{s}}{2}, \quad \beta=\Delta_{s}-\gamma_{\phi}+\frac{\gamma_{s}}{2}
$$

Using the above cubic vertex, one can calculate the corresponding conformal threepoint function by attaching to it the full $\phi$ and $s$ propagators, which are given by,

$$
\langle\phi(x) \phi(0)\rangle=\frac{C_{\phi}\left(1+A_{\phi}\right)}{|x|^{2\left(\Delta_{\phi}+\gamma_{\phi}\right)}}, \quad\langle s(x) s(0)\rangle=\frac{C_{s}\left(1+A_{s}\right)}{|x|^{2\left(\Delta_{s}+\gamma_{s}\right)}}
$$

where the normalizations are,

$$
C_{\phi}=\frac{\Gamma\left(\frac{d}{2}-1\right)}{4 \pi^{\frac{d}{2}}}, \quad C_{s}=\frac{2^{d} \Gamma\left(\frac{d-1}{2}\right) \sin \left(\frac{\pi d}{2}\right)}{\pi^{\frac{3}{2}} \Gamma\left(\frac{d}{2}-2\right)}
$$

and the anomalous dimensions are $[6,7,50,55,61-67]$,

$$
\gamma_{\phi}=\frac{1}{N} \frac{2^{d} \sin \left(\frac{\pi d}{2}\right) \Gamma\left(\frac{d-1}{2}\right)}{\pi^{\frac{3}{2}}(d-2) d \Gamma\left(\frac{d}{2}-2\right)}, \quad \gamma_{s}=\frac{1}{N} \frac{4 \sin \left(\frac{\pi d}{2}\right) \Gamma(d)}{\pi \Gamma\left(\frac{d}{2}+1\right) \Gamma\left(\frac{d}{2}-1\right)}
$$

Note that the integration vertices $x_{1}, x_{2}$ and $x_{3}$ become conformal and can be integrated over using the star-triangle relation (2.12).

We now calculate $\hat{Z}$, up to next-to-leading order in the $1 / N$ expansion. To this end, we notice that the full cubic vertex (A.2) is determined by the sum of the tree level term in (A.1), the counterterm, and the quantum loop corrections. To order $\mathcal{O}\left(1 / N^{\frac{3}{2}}\right)$, it can be represented by the following diagrammatic equation (there is a factor of $-2 / \sqrt{N}$ on both 
sides, which we drop),
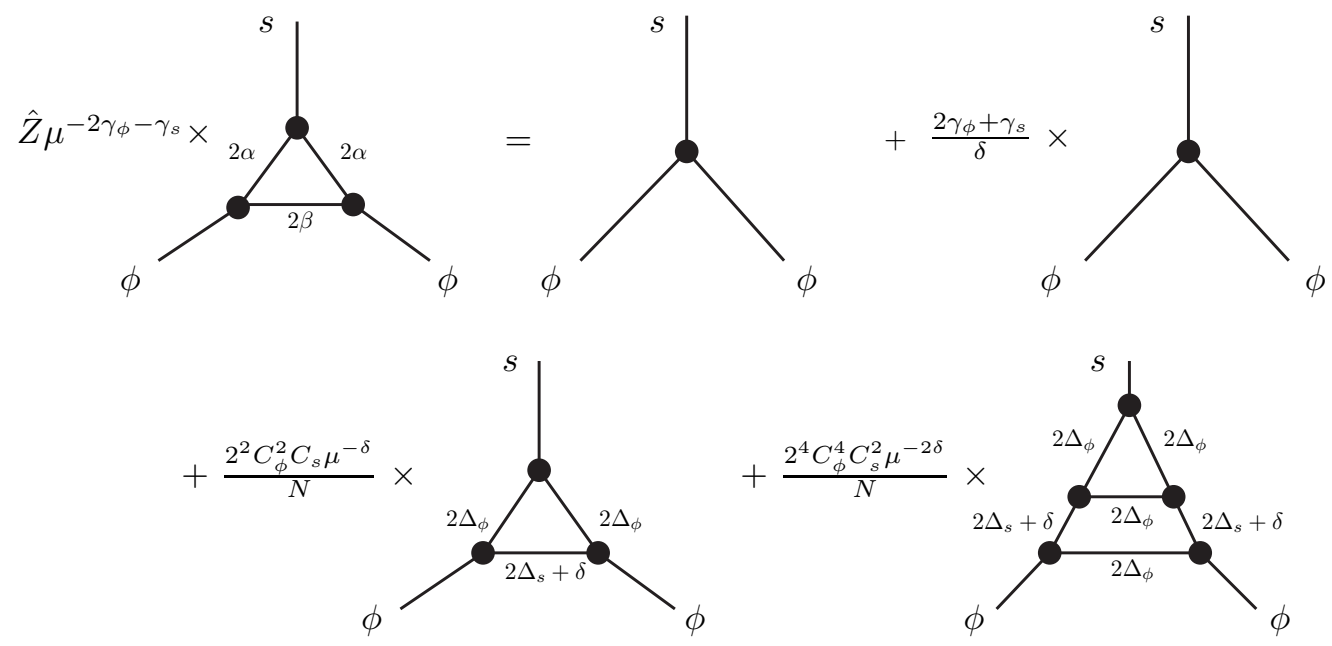

The first term on the right-hand side of (A.7) corresponds to the leading order tree level vertex in (A.1), while the rest of the terms represent $\mathcal{O}\left(1 / N^{\frac{3}{2}}\right)$ corrections. Specifically, the last two diagrams account for the loop corrections to the tree level vertex. The scaling dimension of $s$ in these diagrams undergoes a slight shift of $\delta / 2$, in order to regularize divergent loops. The last graph on the first line of (A.7) is associated with the counterterm, see (A.1); in the limit $\delta \rightarrow 0$, it cancels the divergences of the loop diagrams. The external legs are associated with the fields $\phi$ and $s$, as indicated by the corresponding labels in the figure.

The diagrammatic equation (A.7) defines $\hat{Z}$ up to order $\mathcal{O}\left(1 / N^{\frac{3}{2}}\right)$. It turns out that the background field method simplifies this equation, and getting an explicit expression for $\hat{Z}$ boils down to a relatively short calculation.

To begin with, we decompose the Hubbard-Stratonovich field into a constant background $\bar{s}$ and fluctuating component $s$, i.e. we replace $s \rightarrow \bar{s}+s$. Next, we substitute this decomposition into (A.7) and retain only the linear terms in $\bar{s}$. Now, replacing the external fields $\phi$ with the full propagators (A.4) defines a linear (in $\bar{s}$ ) correction to the two-point function $\left\langle\phi\left(x_{1}\right) \phi\left(x_{2}\right)\right\rangle_{\bar{s}}$, in the presence of a constant background $\bar{s}$,

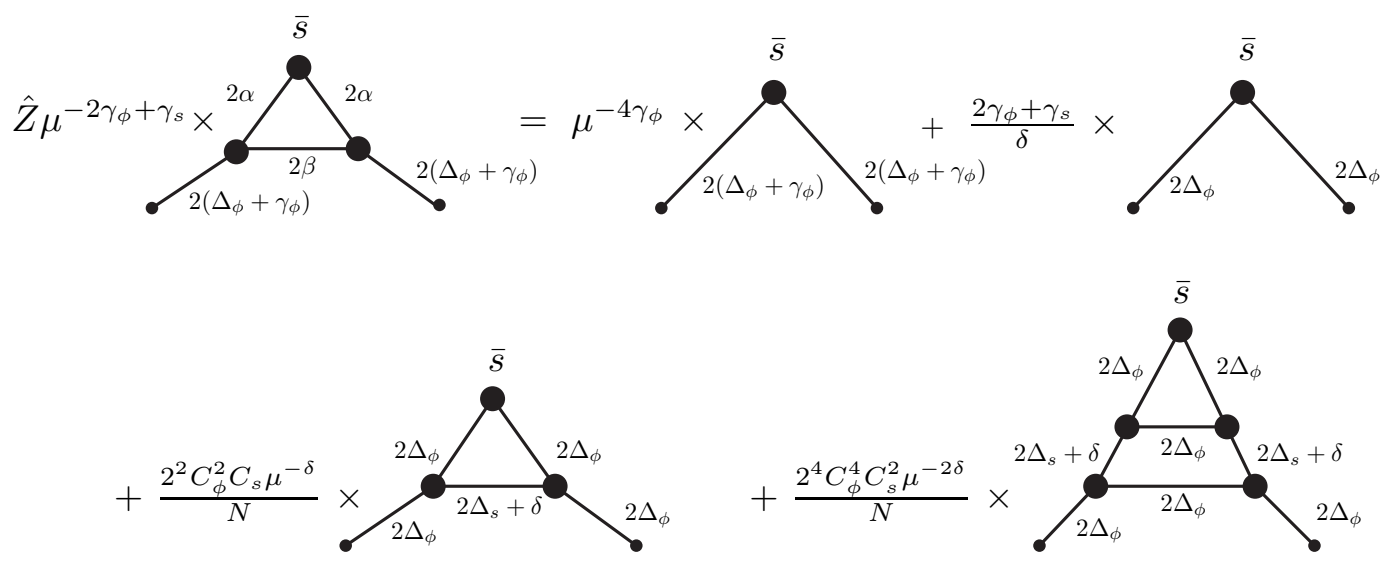


The amplitudes of the full $\phi$-propagators (A.4) cancelled out on both sides of this equation. Furthermore, starting from the integral over the insertion point of the vertex with a constant background $\bar{s}$, all diagrams in (A.8) can be calculated by repeatedly applying the propagator merging relation reviewed in section 4.1.

Carrying out this calculation, one gets for the left-hand side,

$$
\begin{aligned}
\text { l.h.s. of }(\mathrm{A} .8)= & \hat{Z} U\left(\frac{d-\gamma_{s}}{2}-1, \frac{d-\gamma_{s}}{2}-1,2+\gamma_{s}\right) \\
& \times U\left(\frac{d-\gamma_{s}}{2}-\gamma_{\phi}, \frac{d}{2}-1+\gamma_{\phi}, \frac{\gamma_{s}}{2}+1\right) \\
& \times U\left(\frac{d}{2}-1+\gamma_{\phi}, \frac{d-\gamma_{s}}{2}-1,2+\frac{\gamma_{s}}{2}-\gamma_{\phi}\right) \frac{\bar{s} \mu^{-2 \gamma_{\phi}+\gamma_{s}}}{\left|x_{12}\right|^{d-4+2 \gamma_{\phi}-\gamma_{s}}} .
\end{aligned}
$$

Substituting

$$
\hat{Z}=Z_{0}(1+\delta Z),
$$

where $\delta Z=\mathcal{O}(1 / N)$, and expanding in $\gamma_{\phi, s} \sim \mathcal{O}(1 / N)$, yields

$$
\begin{aligned}
\text { l.h.s. of }(\mathrm{A} .8)= & Z_{0} \frac{2(d-2) \pi^{d} U\left(\frac{d}{2}-1, \frac{d}{2}-1,2\right)}{(d-4) \Gamma\left(\frac{d}{2}\right)^{2}\left(2 \gamma_{\phi}+\gamma_{s}\right)} \frac{\bar{s}}{\left|x_{12}\right|^{d-4}}(1+\delta Z+ \\
& \left.\frac{((26-3 d) d-44) \gamma_{s}+2((d-6) d+4) \gamma_{\phi}}{2(d-2)(d-4)}-\left(2 \gamma_{\phi}-\gamma_{s}\right) \log \left(\left|x_{12}\right| \mu\right)\right) .
\end{aligned}
$$

We wish to equate this with the right-hand side of (A.8), which is a sum of four diagrams which we denote by,

$$
\text { r.h.s. of }(\mathrm{A} .8)=v_{\text {tree }}+v_{\text {c.t. }}+v_{\text {loop }}^{(1)}(\delta)+v_{\text {loop }}^{(2)}\left(\frac{\delta}{2}\right) .
$$

Let us evaluate each of the four terms. The tree level term is given by,

$$
v_{\text {tree }}=U\left(\frac{d}{2}-1+\gamma_{\phi}, \frac{d}{2}-1+\gamma_{\phi}, 2-2 \gamma_{\phi}\right) \frac{\bar{s} \mu^{-4 \gamma_{\phi}}}{\left|x_{12}\right|^{d-4+4 \gamma_{\phi}}} .
$$

This term contributes at leading and subleading order in $1 / N$,

$$
\begin{aligned}
v_{\text {tree }}= & U\left(\frac{d}{2}-1, \frac{d}{2}-1,2\right)\left(1+\frac{1}{N} \frac{2^{d-2}(d-6) \sin \left(\frac{\pi d}{2}\right) \Gamma\left(\frac{d-1}{2}\right)}{\pi^{3 / 2} \Gamma\left(\frac{d}{2}+1\right)}-4 \gamma_{\phi} \log \left(\left|x_{12}\right| \mu\right)\right) \\
& \times \frac{\bar{s}}{\left|x_{12}\right|^{d-4}} .
\end{aligned}
$$

The counterterm takes the form,

$$
v_{\text {c.t. }}=U\left(\frac{d}{2}-1, \frac{d}{2}-1,2\right) \frac{2 \gamma_{\phi}+\gamma_{s}}{\delta} \frac{\bar{s}}{\left|x_{12}\right|^{d-4}} .
$$

The first loop diagram is given by,

$$
\begin{aligned}
v_{\text {loop }}^{(1)}(\delta)= & \frac{2^{2} C_{\phi}^{2} C_{s}}{N} \frac{\bar{s}}{\left(\mu\left|x_{12}\right|\right)^{d-4+\delta}} U\left(\frac{d}{2}-1, \frac{d}{2}-1,2\right) U\left(\frac{d+\delta}{2}, \frac{d}{2}-1,1-\frac{\delta}{2}\right) \\
& \times U\left(\frac{d}{2}-1, \frac{d+\delta}{2}-1,2-\frac{\delta}{2}\right) .
\end{aligned}
$$


and the second loop diagram is given by,

$$
\begin{aligned}
v_{\text {loop }}^{(2)}\left(\frac{\delta}{2}\right)= & \frac{2^{4} C_{\phi}^{4} C_{s}^{2}}{N} \frac{\bar{s}}{\left(\mu\left|x_{12}\right|\right)^{d-4+2 \delta}} U\left(\frac{d}{2}-1, \frac{d}{2}-1,2\right) U\left(d-3,2+\frac{\delta}{2}, 1-\frac{\delta}{2}\right) \\
& \times U\left(\frac{d+\delta}{2}-1,2+\frac{\delta}{2}, \frac{d}{2}-1-\delta\right) U\left(\frac{d}{2}-1, \frac{d}{2}+\delta, 1-\delta\right) U\left(\frac{d}{2}-1, \frac{d}{2}-1+\delta, 2-\delta\right) .
\end{aligned}
$$

Expanding (A.16) and (A.17) around $\delta=0$, we obtain,

$$
\begin{aligned}
v_{\text {loop }}^{(1)}(\delta)+v_{\text {loop }}^{(2)}\left(\frac{\delta}{2}\right)= & U\left(\frac{d}{2}-1, \frac{d}{2}-1,2\right) \frac{\bar{s}}{\left|x_{12}\right|^{d-4}} \\
& \times\left(-\frac{2 \gamma_{\phi}+\gamma_{s}}{\delta}+\left(2 \gamma_{\phi}+\gamma_{s}\right) \log \left(\left|x_{12}\right| \mu\right)\right. \\
& \left.\quad+\frac{1}{N} \frac{2^{d-2}(3(d-6) d+28) \sin \left(\frac{\pi d}{2}\right) \Gamma\left(\frac{d-1}{2}\right)}{\pi^{3 / 2}(d-4)(d-2) \Gamma\left(\frac{d}{2}\right)}\right) .
\end{aligned}
$$

Combining (A.14), (A.15) and (A.18), we see that the poles cancel, and we are left with a piece that is finite in the limit $\delta \rightarrow 0$,

$$
\begin{aligned}
v_{\text {tree }}+ & v_{\text {c.t. }}+v_{\text {loop }}^{(1)}(\delta)+v_{\text {loop }}^{(2)}\left(\frac{\delta}{2}\right) \\
\underset{\delta \rightarrow 0}{\longrightarrow} & U\left(\frac{d}{2}-1, \frac{d}{2}-1,2\right) \frac{\bar{s}}{\left|x_{12}\right|^{d-4}} \\
& \times\left(1+\frac{1}{N} \frac{2^{d-3}(d(d(5 d-42)+116)-96) \sin \left(\frac{\pi d}{2}\right) \Gamma\left(\frac{d-1}{2}\right)}{\pi^{3 / 2}(d-4)(d-2) \Gamma\left(\frac{d}{2}+1\right)}-\left(2 \gamma_{\phi}-\gamma_{s}\right) \log \left(\left|x_{12}\right| \mu\right)\right) .
\end{aligned}
$$

Plugging this expression back into (A.12) and comparing to (A.11), yields

$$
\begin{aligned}
Z_{0} & =-\frac{1}{2 \pi^{d}}\left(2 \gamma_{\phi}+\gamma_{s}\right) A(1)^{2} A\left(\frac{d}{2}-2\right) \Gamma\left(\frac{d}{2}\right) \\
\delta Z & =\frac{1}{N} \frac{2^{d-3}(d(d(5 d-42)+116)-96) \sin \left(\frac{\pi d}{2}\right) \Gamma\left(\frac{d-1}{2}\right)}{\pi^{3 / 2}(d-4)(d-2) \Gamma\left(\frac{d}{2}+1\right)} \\
& =\left(\frac{8}{(d-4)^{2}}+\frac{2}{d-2}+\frac{6}{d-4}+5\right) \gamma_{\phi} .
\end{aligned}
$$

This completes the evaluation of the cubic vertex (A.2). This expression matches the result in [55], which was obtained in a different way.

\section{B The $\phi^{6}$ model in terms of auxiliary fields}

In this appendix we give a slight variant of the derivation of some of the results for the sextic model that were presented in the main body of the paper. Recall that the action for the $\phi^{6}$ model is given by $(2.2)$,

$$
S=\int d^{3} x\left(\frac{1}{2}(\partial \phi)^{2}+\frac{1}{\sqrt{N}} \sigma \phi^{2}+\frac{g_{6}}{6 \sqrt{N}} \rho^{3}-\sigma \rho\right) .
$$


Let us explicitly integrate out the $\phi$ fields, which results in the effective action,

$$
S=\frac{N}{2} \operatorname{Tr} \log \left(\partial^{2}-\frac{2 \sigma}{\sqrt{N}}\right)+\int d^{3} x\left(\frac{g_{6}}{6 \sqrt{N}} \rho^{3}-\sigma \rho\right)
$$

We now expand the logarithm to next-to-leading order in $1 / N$. Making use of the matrix element,

$$
\left\langle x\left|\left(\partial^{2}\right)^{-1} \sigma(x)\right| y\right\rangle=-\frac{C_{\phi}}{|x-y|} \sigma(y), \quad C_{\phi}=\frac{1}{4 \pi},
$$

and discarding the constant and linear in $\sigma$ terms (which are removed by appropriate counterterms) we obtain,

$$
\begin{aligned}
S= & \int d^{3} x\left(\frac{g_{6}}{6 \sqrt{N}} \rho^{3}-\sigma \rho\right)-\frac{1}{16 \pi^{2}} \int d^{3} x_{1} d^{3} x_{2} \frac{\sigma\left(x_{1}\right) \sigma\left(x_{2}\right)}{\left|x_{12}\right|^{2}} \\
& +\frac{1}{\sqrt{N}} \frac{1}{48 \pi^{3}} \int d^{3} x_{1} d^{3} x_{2} d^{3} x_{3} \frac{\sigma\left(x_{1}\right) \sigma\left(x_{2}\right) \sigma\left(x_{3}\right)}{\left|x_{12}\right|\left|x_{13}\right|\left|x_{23}\right|}+\mathcal{O}\left(1 / N^{\frac{3}{2}}\right),
\end{aligned}
$$

In order to diagonalize the quadratic part, we define a new field $s$ in terms of $\sigma$,

$$
\sigma(x)=s(x)+\frac{4}{\pi^{2}} \int d^{3} x^{\prime} \frac{\rho\left(x^{\prime}\right)}{\left|x-x^{\prime}\right|^{4}} .
$$

Using the star-triangle relation, as well as the inverse propagator relation,

$$
\int d^{3} x_{2} \frac{1}{\left|x_{12}\right|^{4}\left|x_{23}\right|^{2}}=-2 \pi^{4} \delta^{(3)}\left(x_{13}\right),
$$

we obtain the effective action, up to next-to-leading order,

$$
\begin{aligned}
S= & -\int d^{3} x_{1} d^{3} x_{2}\left(\frac{2}{\pi^{2}} \frac{\rho\left(x_{1}\right) \rho\left(x_{2}\right)}{\left|x_{12}\right|^{4}}+\frac{1}{16 \pi^{2}} \frac{s\left(x_{1}\right) s\left(x_{2}\right)}{\left|x_{12}\right|^{2}}\right) \\
& +\frac{g_{6}}{6 \sqrt{N}} \int d^{3} x_{1} \rho\left(x_{1}\right)^{3}+\frac{1}{\sqrt{N}} \frac{4}{\pi^{2}} \int d^{3} x_{1} d^{3} x_{2} \frac{\rho\left(x_{1}\right)^{2} s\left(x_{2}\right)}{\left|x_{12}\right|^{2}} \\
& +\frac{1}{\sqrt{N}} \frac{4}{3 \pi^{9}} \int \frac{\prod_{i=1}^{3} d^{3} x_{i} d^{3} x_{i}^{\prime}}{\left|x_{1}-x_{1}^{\prime}\right|^{4}\left|x_{2}-x_{2}^{\prime}\right|^{4}\left|x_{3}-x_{3}^{\prime}\right|^{4}} \frac{\rho\left(x_{1}\right) \rho\left(x_{2}\right) \rho\left(x_{3}\right)}{\left|x_{12}^{\prime}\right| x_{13}^{\prime}|| x_{23}^{\prime} \mid} \\
& +\frac{1}{\sqrt{N}} \int \prod_{i=1}^{3} d^{3} x_{i}\left(\frac{1}{48 \pi^{3}} \frac{s\left(x_{1}\right) s\left(x_{2}\right) s\left(x_{3}\right)}{\left|x_{12}\right|\left|x_{13}\right|\left|x_{23}\right|}-\frac{1}{2 \pi^{4}} \frac{\rho\left(x_{1}\right) s\left(x_{2}\right) s\left(x_{3}\right)}{\left|x_{12}\right|^{2}\left|x_{13}\right|^{2}}\right) .
\end{aligned}
$$

Inverting the bilinear part of the action (B.7) through the use of (B.6), we obtain the leading order propagators

$$
\langle\rho(x) \rho(y)\rangle=\frac{1}{8 \pi^{2}} \frac{1}{|x-y|^{2}}, \quad\langle s(x) s(y)\rangle=\frac{4}{\pi^{2}} \frac{1}{|x-y|^{4}} .
$$


The Feynman rules for the amputated cubic vertices are ${ }^{22}$
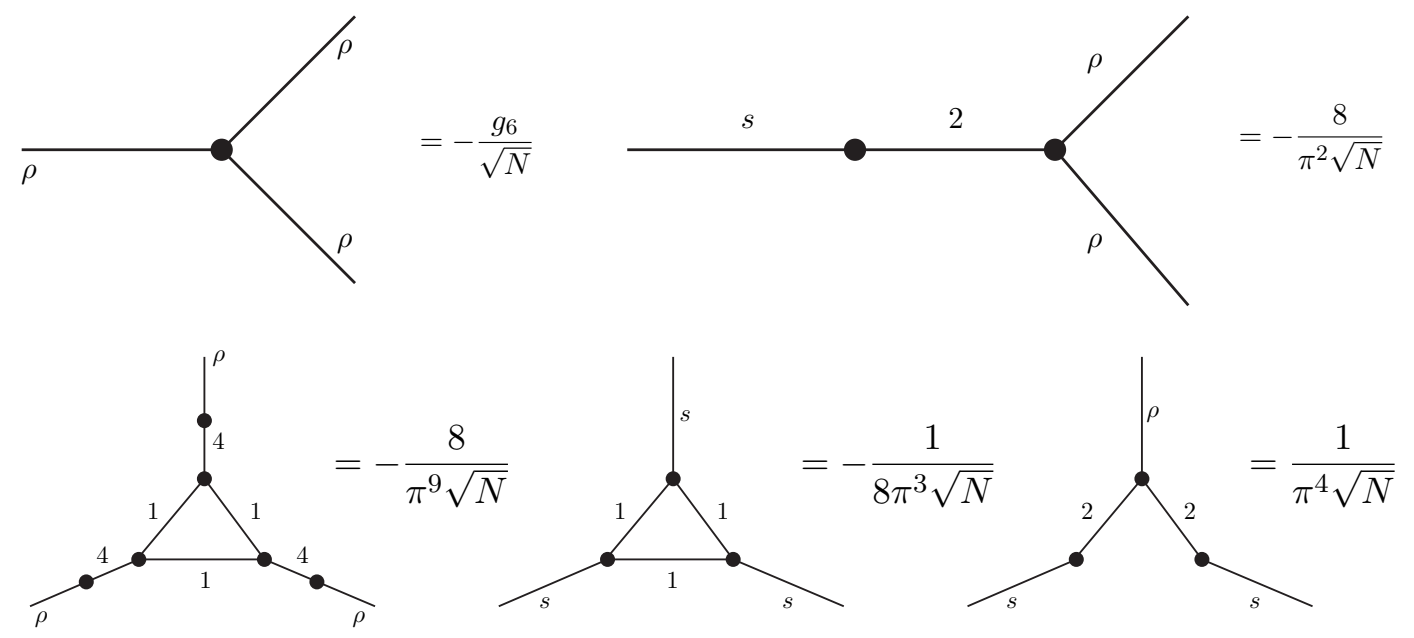

When all three fields $\rho$ are located at different points, we can combine two $\mathcal{O}\left(\rho^{3}\right)$ vertices into one, with the total amplitude $\frac{64-g_{6}}{\sqrt{N}}$. In particular, we recover the leading order threepoint function (3.15).

Using these rules, yields

$$
\begin{aligned}
\left\langle s\left(x_{1}\right) s\left(x_{2}\right) \rho\left(x_{3}\right)\right\rangle & =\mathcal{O}\left(\frac{1}{N^{3 / 2}}\right), \\
\left\langle s\left(x_{1}\right) s\left(x_{2}\right) s\left(x_{3}\right)\right\rangle & =\mathcal{O}\left(\frac{1}{N^{3 / 2}}\right), \\
\left\langle\rho\left(x_{1}\right) \rho\left(x_{2}\right) s\left(x_{3}\right)\right\rangle & =\frac{4}{\pi \sqrt{N}} \frac{1}{\left|x_{13}\right|^{2}\left|x_{23}\right|^{2}}+\mathcal{O}\left(\frac{1}{N^{3 / 2}}\right) .
\end{aligned}
$$

where we used (B.6) and the star-triangle relation, and $s \rightarrow \sqrt{C_{s}} s$.

We conclude this appendix by reproducing the $\rho^{3}$ effective vertex at the next-to-leading order. The contributing diagrams are
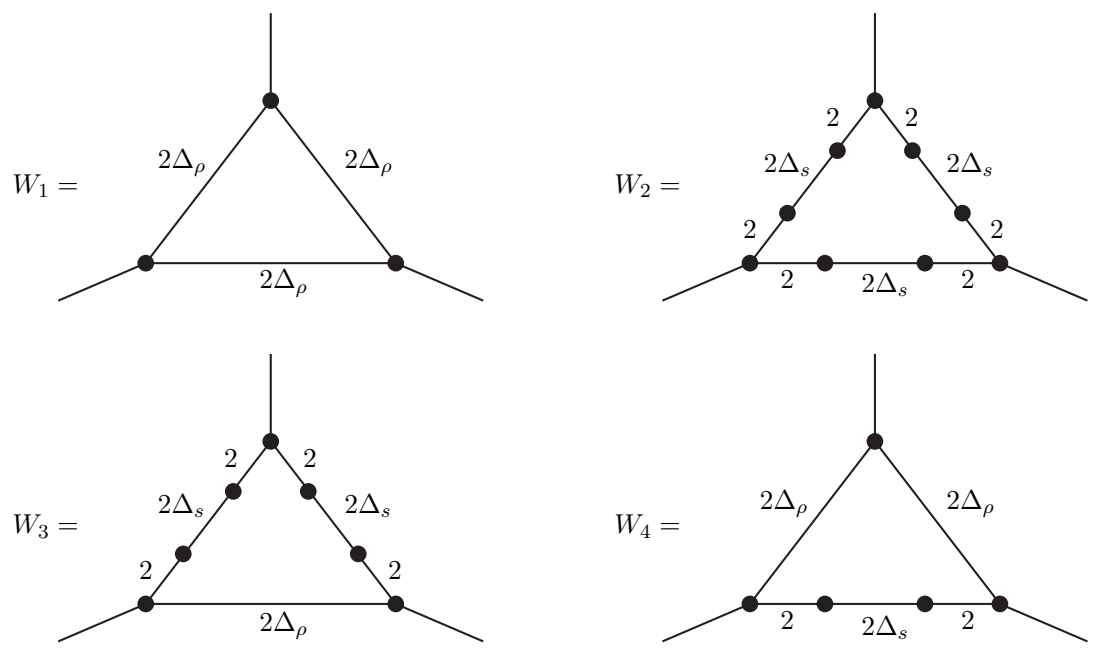

\footnotetext{
${ }^{22}$ It is assumed that all of the three $s$ fields are located at different points $x_{i}, i=1,2,3$, and are connected with the $\phi$-propagator lines. If the locations of any of the two $s$ fields coincide, the corresponding Feynman rule vanishes, owing to the conformal relation $\left\langle\phi^{2}\right\rangle=0$.
} 
Using (B.6), we obtain

$$
\begin{aligned}
& W_{1}=C_{\rho}^{3}\left(\frac{64-g_{6}}{\sqrt{N}}\right)^{3} W_{0}, \\
& W_{2}=C_{s}^{3}\left(\frac{1}{\pi^{4} \sqrt{N}}\right)^{3}\left(-2 \pi^{4}\right)^{3} W_{0}, \\
& W_{3}=3 C_{\rho} C_{s}^{2} \frac{1}{\pi^{4} \sqrt{N}}\left(-\frac{8}{\pi^{2} \sqrt{N}}\right)^{2}\left(-2 \pi^{4}\right)^{2} W_{0}, \\
& W_{4}=3 C_{\rho}^{2} C_{s} \frac{64-g_{6}}{\sqrt{N}}\left(-\frac{8}{\pi^{2} \sqrt{N}}\right)^{2}\left(-2 \pi^{4}\right) W_{0},
\end{aligned}
$$

where $W_{0}=\int d^{3} x_{1,2,3} \frac{\rho\left(x_{1}\right) \rho\left(x_{2}\right) \rho\left(x_{3}\right)}{\left(\left|x_{12}\right|\left|x_{13}\right|\left|x_{23}\right|\right)^{2}}$. The cubic vertex is then given by

$$
\sum_{i=1}^{4} W_{i}=\frac{g_{6}^{2}\left(192-g_{6}\right)}{512 \pi^{6}} \int d^{3} x_{1,2,3} \frac{\rho\left(x_{1}\right) \rho\left(x_{2}\right) \rho\left(x_{3}\right)}{\left(\left|x_{12}\left\|x_{13}\right\| x_{23}\right|\right)^{2}} .
$$

in agreement with (2.14). ${ }^{23}$ This vertex vanishes at the fixed point $g_{6}^{\star}=192$.

Open Access. This article is distributed under the terms of the Creative Commons Attribution License (CC-BY 4.0), which permits any use, distribution and reproduction in any medium, provided the original author(s) and source are credited.

\section{References}

[1] G. Parisi, On self-consistency conditions in conformal covariant field theory, Lett. Nuovo Cim. 4S2 (1972) 777 [inSPIRE].

[2] A.M. Polyakov, Nonhamiltonian approach to conformal quantum field theory, Zh. Eksp. Teor. Fiz. 66 (1974) 23 [Sov. Phys. JETP 39 (1974) 9] [INSPIRE].

[3] S. Ferrara, A.F. Grillo and R. Gatto, Tensor representations of conformal algebra and conformally covariant operator product expansion, Annals Phys. 76 (1973) 161 [INSPIRE].

[4] S. El-Showk, M.F. Paulos, D. Poland, S. Rychkov, D. Simmons-Duffin and A. Vichi, Solving the 3D Ising model with the conformal bootstrap, Phys. Rev. D 86 (2012) 025022 [arXiv: 1203.6064] [INSPIRE].

[5] D. Simmons-Duffin, The conformal bootstrap, arXiv:1602.07982 [INSPIRE].

[6] A.N. Vasiliev, Y.M. Pismak and Y.R. Khonkonen, Simple method of calculating the critical indices in the $1 / N$ expansion, Theor. Math. Phys. 46 (1981) 104 [Teor. Mat. Fiz. 46 (1981) 157] [INSPIRE].

[7] A.N. Vasiliev, Y.M. Pismak and Y.R. Khonkonen, $1 / N$ expansion: calculation of the exponents $\eta$ and $\nu$ in the order $1 / N^{2}$ for arbitrary number of dimensions, Theor. Math. Phys. 47 (1981) 465 [Teor. Mat. Fiz. 47 (1981) 291] [inSPIRE].

[8] G. 't Hooft, A planar diagram theory for strong interactions, Nucl. Phys. B 72 (1974) 461 [INSPIRE].

[9] E. Witten, Baryons in the $1 / N$ expansion, Nucl. Phys. B 160 (1979) 57.

\footnotetext{
${ }^{23}$ Recall that effective vertex and the corresponding diagram are related by the symmetry factor of $-1 / 3$ !
} 
[10] K.G. Wilson and M.E. Fisher, Critical exponents in 3.99 dimensions, Phys. Rev. Lett. 28 (1972) 240 [INSPIRE].

[11] T. Banks and A. Zaks, On the phase structure of vector-like gauge theories with massless fermions, Nucl. Phys. B 196 (1982) 189.

[12] A.D. Bond and D.F. Litim, Price of asymptotic safety, Phys. Rev. Lett. 122 (2019) 211601 [arXiv: 1801.08527] [INSPIRE].

[13] G. Parisi, The theory of nonrenormalizable interactions. 1. The large $N$ expansion, Nucl. Phys. B 100 (1975) 368 [inSPIRE].

[14] D.J. Gross and A. Neveu, Dynamical symmetry breaking in asymptotically free field theories, Phys. Rev. D 10 (1974) 3235 [inSPIRE].

[15] J. Zinn-Justin, Four fermion interaction near four-dimensions, Nucl. Phys. B 367 (1991) 105 [InSPIRE].

[16] M.E. Fisher, Yang-Lee edge singularity and $\phi^{3}$ field theory, Phys. Rev. Lett. 40 (1978) 1610 [INSPIRE].

[17] L. Fei, S. Giombi and I.R. Klebanov, Critical $O(N)$ models in $6-\epsilon$ dimensions, Phys. Rev. D 90 (2014) 025018 [arXiv:1404.1094] [INSPIRE].

[18] L. Fei, S. Giombi, I.R. Klebanov and G. Tarnopolsky, Three loop analysis of the critical $O(N)$ models in $6-\varepsilon$ dimensions, Phys. Rev. D 91 (2015) 045011 [arXiv:1411.1099] [INSPIRE].

[19] S. Sachdev and J. Ye, Gapless spin-fluid ground state in a random quantum Heisenberg magnet, Phys. Rev. Lett. 70 (1993) 3339.

[20] A. Kitaev, A simple model of quantum holography, talks given at KITP, April 7 and May 27 (2015).

[21] J. Maldacena and D. Stanford, Remarks on the Sachdev-Ye-Kitaev model, Phys. Rev. D 94 (2016) 106002 [arXiv:1604.07818] [inSPIRE].

[22] D.J. Gross and V. Rosenhaus, All point correlation functions in SYK, JHEP 12 (2017) 148 [arXiv: 1710.08113] [INSPIRE].

[23] V. Rosenhaus, An introduction to the SYK model, J. Phys. A 52 (2019) 323001 [arXiv: 1807.03334] [INSPIRE].

[24] S. Giombi, I.R. Klebanov and G. Tarnopolsky, Bosonic tensor models at large $N$ and small $\epsilon$, Phys. Rev. D 96 (2017) 106014 [arXiv:1707.03866] [INSPIRE].

[25] I.R. Klebanov, F. Popov and G. Tarnopolsky, TASI lectures on large $N$ tensor models, PoS (TASI2017) 004 [arXiv: 1808.09434] [INSPIRE].

[26] R.D. Pisarski, Fixed point structure of $\left(\phi^{6}\right)$ in three-dimensions at large $N$, Phys. Rev. Lett. 48 (1982) 574 [INSPIRE].

[27] D.F. Litim and E. Marchais, Critical $O(N)$ models in the complex field plane, Phys. Rev. D 95 (2017) 025026 [arXiv: 1607.02030] [InSPIRE].

[28] A. Jüttner, D.F. Litim and E. Marchais, Global Wilson-Fisher fixed points, Nucl. Phys. B 921 (2017) 769 [arXiv:1701.05168] [INSPIRE].

[29] J. Goldstone, A. Salam and S. Weinberg, Broken symmetries, Phys. Rev. 127 (1962) 965 [INSPIRE].

[30] W.A. Bardeen, M. Moshe and M. Bander, Spontaneous breaking of scale invariance and the ultraviolet fixed point in $O(N)$ symmetric ( $\bar{\phi}_{3}^{6}$ in three-dimensions) theory, Phys. Rev. Lett. 52 (1984) 1188 [INSPIRE]. 
[31] E. Rabinovici, B. Saering and W.A. Bardeen, Critical surfaces and flat directions in a finite theory, Phys. Rev. D 36 (1987) 562 [InSPIRE].

[32] D.F. Litim, E. Marchais and P. Mati, Fixed points and the spontaneous breaking of scale invariance, Phys. Rev. D 95 (2017) 125006 [arXiv:1702.05749] [INSPIRE].

[33] C. Fleming, B. Delamotte and S. Yabunaka, Finite $N$ origin of the Bardeen-Moshe-Bander phenomenon and its extension at $N=\infty$ by singular fixed points, Phys. Rev. D 102 (2020) 065008 [arXiv: 2001. 07682] [INSPIRE].

[34] J.A. Gracey, Calculation of exponent $\eta$ to $O\left(1 / N^{2}\right)$ in the $O(N)$ Gross-Neveu model, Int. J. Mod. Phys. A 6 (1991) 395 [Erratum ibid. 6 (1991) 2755] [INSPIRE].

[35] J.A. Gracey, Anomalous mass dimension at $O\left(1 / N^{2}\right)$ in the $O(N)$ Gross-Neveu model, Phys. Lett. B 297 (1992) 293 [INSPIRE].

[36] S.E. Derkachov, N.A. Kivel, A.S. Stepanenko and A.N. Vasiliev, On calculation in $1 / n$ expansions of critical exponents in the Gross-Neveu model with the conformal technique, hep-th/9302034 [INSPIRE].

[37] A.N. Vasiliev, S.E. Derkachov, N.A. Kivel and A.S. Stepanenko, The $1 / n$ expansion in the Gross-Neveu model: conformal bootstrap calculation of the index eta in order $1 / n^{3}$, Theor. Math. Phys. 94 (1993) 127 [Teor. Mat. Fiz. 94 (1993) 179] [InSPIRE].

[38] A.N. Vasiliev and A.S. Stepanenko, The $1 / n$ expansion in the Gross-Neveu model: Conformal bootstrap calculation of the exponent $1 / \nu$ to the order $1 / n^{2}$, Theor. Math. Phys. 97 (1993) 1349 [Teor. Mat. Fiz. 97 (1993) 364] [INSPIRE].

[39] J.A. Gracey, Computation of beta-prime $\left(g_{c}\right)$ at $O\left(1 / N^{2}\right)$ in the $O(N)$ Gross-Neveu model in arbitrary dimensions, Int. J. Mod. Phys. A 9 (1994) 567 [hep-th/9306106] [InSPIRE].

[40] J.A. Gracey, Computation of critical exponent eta at $O\left(1 / N^{3}\right)$ in the four Fermi model in arbitrary dimensions, Int. J. Mod. Phys. A 9 (1994) 727 [hep-th/9306107] [INSPIRE].

[41] A.N. Manashov and E.D. Skvortsov, Higher-spin currents in the Gross-Neveu model at $1 / n^{2}$, JHEP 01 (2017) 132 [arXiv:1610.06938] [INSPIRE].

[42] A.N. Manashov and M. Strohmaier, Correction exponents in the Gross-Neveu-Yukawa model at $1 / N^{2}$, Eur. Phys. J. C $\mathbf{7 8}$ (2018) 454 [arXiv:1711.02493] [inSPIRE].

[43] J.A. Gracey, T. Luthe and Y. Schröder, Four loop renormalization of the Gross-Neveu model, Phys. Rev. D 94 (2016) 125028 [arXiv: 1609.05071] [INSPIRE].

[44] M. Preti, STR: a Mathematica package for the method of uniqueness, Int. J. Mod. Phys. C 31 (2020) 2050146 [arXiv:1811.04935] [INSPIRE].

[45] A.N. Vasiliev and M.Y. Nalimov, Analog of dimensional regularization for calculation of the renormalization group functions in the $1 / n$ expansion for arbitrary dimension of space, Theor. Math. Phys. 55 (1983) 423 [Teor. Mat. Fiz. 55 (1983) 163] [InSPIRE].

[46] M. Moshe and J. Zinn-Justin, Quantum field theory in the large $N$ limit: a review, Phys. Rept. 385 (2003) 69 [hep-th/0306133] [INSPIRE].

[47] K. Diab, L. Fei, S. Giombi, I.R. Klebanov and G. Tarnopolsky, $O n C_{J}$ and $C_{T}$ in the Gross-Neveu and $O(N)$ models, J. Phys. A 49 (2016) 405402 [arXiv:1601.07198] [InSPIRE].

[48] L. Fei, S. Giombi, I.R. Klebanov and G. Tarnopolsky, Yukawa CFTs and emergent supersymmetry, PTEP 2016 (2016) 12C105 [arXiv: 1607.05316] [INSPIRE].

[49] J.A. Gracey, Four loop renormalization of $\phi^{3}$ theory in six dimensions, Phys. Rev. D 92 (2015) 025012 [arXiv:1506.03357] [INSPIRE]. 
[50] M. Goykhman and M. Smolkin, Vector model in various dimensions, Phys. Rev. D 102 (2020) 025003 [arXiv: 1911.08298] [INSPIRE].

[51] S. Weinberg, Six-dimensional methods for four-dimensional conformal field theories, Phys. Rev. D 82 (2010) 045031 [arXiv: 1006.3480] [INSPIRE].

[52] M. Goykhman, T. Shachar and M. Smolkin, On fast quenches and spinning correlators, JHEP 06 (2018) 168 [arXiv:1804.03855] [INSPIRE].

[53] M. Goykhman and R. Sinha, CFT data in the Gross-Neveu model, arXiv:2011.07768 [INSPIRE].

[54] A.M. Polyakov, Conformal symmetry of critical fluctuations, JETP Lett. 12 (1970) 381 [Pisma Zh. Eksp. Teor. Fiz. 12 (1970) 538] [INSPIRE].

[55] S.E. Derkachov and A.N. Manashov, The simple scheme for the calculation of the anomalous dimensions of composite operators in the $1 / N$ expansion, Nucl. Phys. B 522 (1998) 301 [hep-th/9710015] [INSPIRE].

[56] B. Craps, T. Hertog and N. Turok, A multitrace deformation of ABJM theory, Phys. Rev. D 80 (2009) 086007 [arXiv:0905.0709] [InSPIRE].

[57] M. Smolkin and N. Turok, Dual description of a $4 d$ cosmology, arXiv:1211.1322 [INSPIRE].

[58] B. Craps, T. Hertog and N. Turok, On the quantum resolution of cosmological singularities using AdS/CFT, Phys. Rev. D 86 (2012) 043513 [arXiv:0712.4180] [INSPIRE].

[59] O. Aharony, O. Bergman, D.L. Jafferis and J. Maldacena, $N=6$ superconformal Chern-Simons-matter theories, M2-branes and their gravity duals, JHEP 10 (2008) 091 [arXiv: 0806.1218] [INSPIRE].

[60] N. Chai, S. Chaudhuri, C. Choi, Z. Komargodski, E. Rabinovici and M. Smolkin, Thermal order in conformal theories, Phys. Rev. D 102 (2020) 065014 [arXiv:2005.03676] [INSPIRE].

[61] K. Lang and W. Rühl, Critical nonlinear $O(N) \sigma$-models at $2<d<4$ : the degeneracy of quasiprimary fields and it resolution, Z. Phys. C 61 (1994) 495 [INSPIRE].

[62] A.C. Petkou, $C(T)$ and $C(J)$ up to next-to-leading order in $1 / N$ in the conformally invariant $O(N)$ vector model for $2<d<4$, Phys. Lett. B 359 (1995) 101 [hep-th/9506116] [InSPIRE].

[63] A. Petkou, Conserved currents, consistency relations and operator product expansions in the conformally invariant $O(N)$ vector model, Annals Phys. 249 (1996) 180 [hep-th/9410093] [INSPIRE].

[64] T. Leonhardt and W. Rühl, The minimal conformal $O(N)$ vector $\sigma$-model at $d=3$, J. Phys. A 37 (2004) 1403 [hep-th/0308111] [InSPIRE].

[65] J.A. Gracey, Large $N_{f}$ quantum field theory, Int. J. Mod. Phys. A 33 (2019) 1830032 [arXiv: 1812.05368] [INSPIRE].

[66] L.F. Alday, J. Henriksson and M. van Loon, An alternative to diagrams for the critical $O(N)$ model: dimensions and structure constants to order $1 / N^{2}$, JHEP 01 (2020) 063 [arXiv: 1907.02445] [INSPIRE].

[67] S. Giombi, R. Huang, I.R. Klebanov, S.S. Pufu and G. Tarnopolsky, The $O(N)$ model in $4<d<6$ : instantons and complex CFTs, Phys. Rev. D 101 (2020) 045013 [arXiv: 1910.02462] [INSPIRE]. 